\title{
HARMONIC ALMOST HERMITIAN STRUCTURES
}

\author{
JOHANN DAVIDOV
}

\begin{abstract}
This is a survey of old and new results on the problem when a compatible almost complex structure on a Riemannian manifold is a harmonic section or a harmonic map from the manifold into its twistor space. In this context, special attention is paid to the Atiyah-Hitchin-Singer and Eells-Salamon almost complex structures on the twistor space of an oriented Riemannian four-manifold.

2010 Mathematics Subject Classification. Primary 53C43, Secondary 58E20, $53 \mathrm{C} 28$

Key words: almost complex structures, twistor spaces, harmonic maps
\end{abstract}

\section{INTRODUCTION}

Recall that an almost complex structure on a Riemannian manifold $(N, h)$ is called almost Hermitian (or, compatible) if it is $h$-orthogonal. If a Riemannian manifold admits an almost Hermitian structure, it possesses many such structures (cf. Section 3). Thus, it is natural to look for "reasonable" criteria that distinguish some of these structures. A natural way to obtain such criteria is to consider the almost Hermitian structures on $(N, h)$ as sections of its twistor bundle $\pi: \mathcal{T} \rightarrow$ $N$ whose fibre at a point $p \in N$ consists of all $h$-orthogonal complex structures $J_{p}: T_{p} N \rightarrow T_{p} N$ on the tangent space of $N$ at $p$. The fibre of the bundle $\mathcal{T}$ is the compact Hermitian symmetric space $O(2 m) / U(m), 2 m=\operatorname{dim} N$, and its standard metric $-\frac{1}{2}$ Trace $J_{1} \circ J_{2}$ is Kähler-Einstein. The twistor space $\mathcal{T}$ admits a natural Riemannian metric $h_{1}$ such that the projection map $\pi:\left(\mathcal{T}, h_{1}\right) \rightarrow(N, h)$ is a Riemannian submersion with totally geodesic fibres. This metric is compatible with the natural almost complex structures on $\mathcal{T}$, which have been introduced by Atiyah-Hitchin-Singer 2 and Eells-Salamon $[16$ in the case $\operatorname{dim} N=4$.

If $N$ is oriented, the twistor space $\mathcal{T}$ has two connected components often called positive and negative twistor spaces of $(N, h)$; their sections are almost Hermitian structures yielding the orientation and, respectively, the opposite orientation of $N$.

E. Calabi and H. Gluck [6] have proposed to single out those almost Hermitian structures $J$ on $(N, h)$, whose image $J(N)$ in $\mathcal{T}$ is of minimal volume with respect to the metric $h_{1}$. They have proved that the standard almost Hermitian structure on the 6 -sphere $S^{6}$, defined by means of the Cayley numbers, can be characterized by that property.

Motivated by harmonic map theory, C. M. Wood [35, 36, has suggested to consider as "optimal" those almost-Hermitian structures $J:(N, h) \rightarrow\left(\mathcal{T}, h_{1}\right)$, which are critical points of the energy functional under variations through sections of $\mathcal{T}$.

The author is partially supported by the National Science Fund, Ministry of Education and Science of Bulgaria under contract DFNI-I 02/14. 
In general, these critical points are not harmonic maps, but, by analogy, in 36. they are referred to as "harmonic almost complex structures". They are also called "harmonic sections" 35], a term, which is more appropriate in the context of this article.

Forgetting the bundle structure of $\mathcal{T}$, we can consider almost Hermitian structures that are critical points of the energy functional under variations through all maps $N \rightarrow \mathcal{T}$. These structures are genuine harmonic maps from $(N, h)$ into $\left(\mathcal{T}, h_{1}\right)$ and we refer to [15] for basic fact about such maps.

The main goal of this paper is to survey results about the harmonicity (in both senses) of the Atiyah-Hitchin-Singer and Eells-Salamon almost Hermitian structures on the twistor space of an oriented four-dimensional Riemannian manifold, as well as almost Hermitian structures on such a manifold.

In Section 2 we recall some basic facts about the twistor spaces of even-dimensional Riemannian manifolds. Special attention is paid to the twistor spaces of oriented four-dimensional manifolds. In Sections 3 and 4 we discuss the energy functional on sections of a twistor space, i.e. almost Hermitian structures on the base Riemannian manifold. We state the Euler-Lagrange equation for such a structure to be a critical point of the energy functional (a harmonic section) obtained by Wood [35, 36. Several examples of non-Kähler almost Hermitian structures, which are harmonic sections are given. Kähler structures are absolute minima of the energy functional. G. Bor, L. Hernández-Lamoneda and M. Salvai 4 have given sufficient conditions for an almost Hermitian structure to be a minimizer of the energy functional. Their result (in fact, part of it) is presented in Section 4 and is used to supply examples of non-Kähler minimizers based on works by C. LeBrun 27] and I. Kim 24. Section 4 ends with a lemma from [9, which rephrases the Euler-Lagrange equation for an almost Hermitian structure $(h, J)$ on a manifold $N$ in terms of the fundamental 2 -form of $(h, J)$ and the curvature of $(N, h)$. This lemma has been used in [9] to show that the Atiyah-Hitchin-Singer almost Hermitian structure $\mathcal{J}_{1}$ on the negative twistor space $\mathcal{Z}$ of an oriented Riemannian 4-manifold $(M, g)$ is a harmonic section if and only if the base manifold $(M, g)$ is self-dual, while the Eells-Salamon structure $\mathcal{J}_{2}$ is a harmonic section if and only $(M, g)$ is self-dual and of constant scalar curvature. The main part of the proof of this result (slightly different from the proof in [9]) is presented in Section 5. In this context, it is natural to ask when $\mathcal{J}_{1}$ and $\mathcal{J}_{2}$ are harmonic maps into the twistor space of $\mathcal{Z}$. Recall that a map between Riemannian manifolds is harmonic if and only if the trace of its second fundamental form vanishes. Section 6 contains a computation of the second fundamental form of a map from a Riemannian manifold $(N, h)$ into its twistor space $\left(\mathcal{T}, h_{1}\right)$. The corresponding formula obtained in this section is used in Section 7 to give an answer to the question above: $\mathcal{J}_{1}$ or $\mathcal{J}_{2}$ is a harmonic map if and only if $(M, g)$ is either self-dual and Einstein, or locally is the product of an open interval on $\mathbb{R}$ and a 3-dimensional Riemannian manifold of constant curvature. A sketch of the proof, involving the main theorem of Section 5 and several technical lemmas, is given in Section 7 following [11. In Section 8 we give geometric conditions on a fourdimensional almost Hermitian manifold $(M, g, J)$ under which the almost complex structure $J$ is a harmonic map of $(M, g)$ into the positive twistor space $\left(\mathcal{Z}_{+}, h_{1}\right)$, $M$ being considered with the orientation induced by $J$. We also find conditions for minimality of the submanifold $J(M)$ of the twistor space $\mathcal{Z}_{+}$. As is well-known, in dimension four, there are three basic classes in the Gray-Hervella classification [19] 
of almost Hermitian structures: Hermitian, almost Kähler (symplectic) and Kähler structures. As for a manifold of an arbitrary dimension, if $(g, J)$ is Kähler, the map $J:(M, g) \rightarrow\left(\mathcal{Z}_{+}, h_{1}\right)$ is a totally geodesic isometric imbedding. In the case of a Hermitian structure, we express the conditions for harmonicity or minimality of $J$ in terms of the Lee form, the Ricci and star-Ricci tensors of $(M, g, J)$, while for an almost Kähler structure the conditions are in terms of the Ricci, star-Ricci and Nijenhuis tensors. Several examples illustrating these results are discussed at the end of Section 8, among them a Hermitian structure that is a harmonic section of the twistor bundle $\mathcal{Z}_{+}$and a minimal isometric imbedding in it, but not a harmonic map.

Acknowledgment. This paper is an expanded version on the author's talk at the INdAM workshop in Rome, November, 16-20, 2015, on the occasion of the sixtieth birthday of Simon Salamon. The author would like to express his gratitude to the organizers and Simon for the invitation to take part in the workshop and for the wonderful and stimulating environment surrounding it. Special thanks are also due to the referee whose remarks helped to improve the final version of the article.

\section{The twistor space of An EVEn-Dimensional RiEmannian manifold}

Denote by $F\left(\mathbb{R}^{2 m}\right)$ the set of complex structures on $\mathbb{R}^{2 m}$ compatible with its standard metric $g$. This set has the structure of an imbedded submanifold of the vector space $s o(2 m)$ of skew-symmetric endomorphisms of $\mathbb{R}^{2 m}$. The tangent space of $F\left(\mathbb{R}^{2 m}\right)$ at a point $J$ consists of all skew-symmetric endomorphisms of $\mathbb{R}^{2 m}$ anti-commuting with $J$. Then we can define an almost complex structure on the manifold $F\left(\mathbb{R}^{2 m}\right)$ setting

$$
\mathcal{J} Q=J Q \text { for } Q \in T_{J} F\left(\mathbb{R}^{2 m}\right) .
$$

This almost complex structure is compatible with the standard metric

$$
G(A, B)=-\frac{1}{2 m} \text { Trace } A B
$$

of $s o(2 m)$, where the factor $1 / 2 m$ is chosen so that every $J \in F\left(\mathbb{R}^{2 m}\right)$ should have unit norm. In fact, the almost Hermitian structure $(G, \mathcal{J})$ is Kähler-Einstein.

The group $O(2 m)$ acts on $F\left(\mathbb{R}^{2 m}\right)$ by conjugation and the isotropy subgroup at the standard complex structure $J_{0}$ of $\mathbb{R}^{2 m} \cong \mathbb{C}^{m}$ is $U(m)$. Therefore $F\left(\mathbb{R}^{2 m}\right)$ can be identified with the homogeneous space $O(2 m) / U(m)$.

Note also that the manifold $F\left(\mathbb{R}^{2 m}\right)$ has two connected components: if we fix an orientation on $\mathbb{R}^{2 m}$, these components consists of all complex structures on $\mathbb{R}^{2 m}$ compatible with the metric $g$ and inducing \pm the orientation of $\mathbb{R}^{2 m}$; each of them has the homogeneous representation $S O(2 m) / U(m)$.

The twistor space of an even-dimensional Riemannian manifold $(N, h), \operatorname{dim} N=$ $2 m$, is the bundle $\pi: \mathcal{T} \rightarrow N$, whose fibre at every point $p \in N$ is the space of compatible complex structures on the Euclidean vector space $\left(T_{p} N, h_{p}\right)$. This is the associated bundle

$$
\mathcal{T}=O(N) \times_{O(2 m)} F\left(\mathbb{R}^{2 m}\right)
$$

where $O(N)$ is the principal bundle of orthonormal frames on $N$. Since the bundle $\pi: \mathcal{T} \rightarrow N$ is associated to $O(N)$, the Levi-Civita connection on $(N, h)$ gives rise to a splitting $\mathcal{V} \oplus \mathcal{H}$ of the tangent bundle of the manifold $\mathcal{T}$. Using this splitting, we can define a natural 1-parameter family of Riemannian metrics $h_{t}, t>0$, as follows. 
For every $J \in \mathcal{T}$, the horizontal subspace $\mathcal{H}_{J}$ of $T_{J} \mathcal{T}$ is isomorphic to the tangent space $T_{\pi(J)} N$ via the differential $\pi_{* J}$ and the metric $h_{t}$ on $\mathcal{H}_{J}$ is the lift of the metric $h$ on $T_{\pi(J)} N, h_{t} \mid \mathcal{H}_{J}=\pi^{*} h$. The vertical subspace $\mathcal{V}_{J}$ of $T_{J} \mathcal{T}$ is the tangent space at $J$ to the fibre through $J$ of the bundle $\mathcal{T}$ and $h_{t} \mid \mathcal{V}_{J}$ is defined as $t$ times the metric $G$ of this fibre. Finally, the horizontal space $\mathcal{H}_{J}$ and the vertical space $\mathcal{V}_{J}$ are declared to be orthogonal. Then, by the Vilms theorem [34, the projection $\pi:\left(\mathcal{T}, h_{t}\right) \rightarrow(N, h)$ is a Riemannian submersion with totally geodesic fibres (this can also be proved directly).

It is often convenient to consider $\mathcal{T}$ as a submanifold of the bundle

$$
\pi: A(T N)=O(N) \times_{O(2 m)} s o(2 m) \rightarrow N
$$

of skew-symmetric endomorphisms of $T N$. The inclusion of $\mathcal{T}$ into $A(T N)$ is fibrepreserving and for every $J \in \mathcal{T}$ the horizontal subspace $\mathcal{H}_{J}$ of $T_{J} \mathcal{T}$ coincides with the horizontal subspace of $T_{J} A(T N)$ with respect to the connection induced by the Levi-Civita connection of $(N, h)$ since the inclusion of $F\left(\mathbb{R}^{2 m}\right)$ into $s o(2 m)$ is $O(2 m)$-equivariant; the vertical subspace $\mathcal{V}_{J}$ of $T_{J} \mathcal{T}$ is the subspace of the fibre $A\left(T_{\pi(J)} N\right)$ of $A(T N)$ through $J$ consisting of the skew-symmetric endomorphisms of $T_{\pi(J)} N$ anti-commuting with $J$.

If the manifold $N$ is oriented, its twistor space has two connected components, the spaces of compatible complex structures on tangent spaces of $N$ yielding the given, or the opposite orientation of $N$. These are often called the positive, respectively, the negative twistor space.

2.1. The twistor space of an oriented four-dimensional Riemannian manifold. In dimension four, each of the two connected components $F\left(\mathbb{R}^{4}\right)$ can be identified with the unit sphere $S^{2}$. It is often convenient to describe this identification in terms of the space $\Lambda^{2} \mathbb{R}^{4}$. The metric $g$ of $\mathbb{R}^{4}$ induces a metric on $\Lambda^{2} \mathbb{R}^{4}$ given by

$$
g\left(x_{1} \wedge x_{2}, x_{3} \wedge x_{4}\right)=\frac{1}{2}\left[g\left(x_{1}, x_{3}\right) g\left(x_{2}, x_{4}\right)-g\left(x_{1}, x_{4}\right) g\left(x_{2}, x_{3}\right)\right],
$$

the factor $1 / 2$ being chosen in consistency with 8 , 9. Consider the isomorphisms so(4) $\cong \Lambda^{2} \mathbb{R}^{4}$ sending $\varphi \in \operatorname{so}(4)$ to the 2 -vector $\varphi^{\wedge}$ for which

$$
2 g\left(\varphi^{\wedge}, x \wedge y\right)=g(\varphi x, y), \quad x, y \in \mathbb{R}^{4} .
$$

This isomorphism is an isometry with respect to the metric $G$ on $s o(4)$ and the metric $g$ on $\Lambda^{2} \mathbb{R}^{4}$. Given $a \in \Lambda^{2} \mathbb{R}^{4}$, the skew-symmetric endomorphism of $\mathbb{R}^{4}$ corresponding to $a$ under the inverse isomorphism will be denoted by $K_{a}$.

Fix an orientation on $\mathbb{R}^{4}$ and denote by $F_{ \pm}\left(\mathbb{R}^{4}\right)$ the set of complex structures on $\mathbb{R}^{4}$ compatible with the metric $g$ and inducing \pm the orientation of $\mathbb{R}^{4}$.

The Hodge star operator defines an endomorphism $*$ of $\Lambda^{2} \mathbb{R}^{4}$ with $*^{2}=I d$. Hence we have the orthogonal decomposition

$$
\Lambda^{2} \mathbb{R}^{4}=\Lambda_{-}^{2} \mathbb{R}^{4} \oplus \Lambda_{+}^{2} \mathbb{R}^{4},
$$

where $\Lambda_{ \pm}^{2} \mathbb{R}^{4}$ are the subspaces of $\Lambda^{2} \mathbb{R}^{4}$ corresponding to the $( \pm 1)$-eigenvalues of the operator $*$. Let $\left(e_{1}, e_{2}, e_{3}, e_{4}\right)$ be an oriented orthonormal basis of $\mathbb{R}^{4}$. Set

(2) $s_{1}^{ \pm}=e_{1} \wedge e_{2} \pm e_{3} \wedge e_{4}, \quad s_{2}^{ \pm}=e_{1} \wedge e_{3} \pm e_{4} \wedge e_{2}, \quad s_{3}^{ \pm}=e_{1} \wedge e_{4} \pm e_{2} \wedge e_{3}$.

Then $\left(s_{1}^{ \pm}, s_{2}^{ \pm}, s_{3}^{ \pm}\right)$is an orthonormal basis of $\Lambda_{ \pm}^{2} \mathbb{R}^{4}$.

It is easy to see that the isomorphism $\varphi \rightarrow \varphi^{\wedge}$ identifies $F_{ \pm}\left(\mathbb{R}^{4}\right)$ with the unit sphere $S\left(\Lambda_{ \pm}^{2} \mathbb{R}^{4}\right)$ of the Euclidean vector space $\left(\Lambda_{ \pm}^{2} \mathbb{R}^{n}, g\right)$. Under this isomorphism, 
if $J \in F_{ \pm}\left(\mathbb{R}^{4}\right)$, the tangent space $T_{J} F\left(\mathbb{R}^{4}\right)=T_{J} F_{ \pm}\left(\mathbb{R}^{4}\right)$ is identified with the orthogonal complement $(\mathbb{R} J)^{\perp}$ of the space $\mathbb{R} J$ in $\Lambda_{ \pm}^{2} \mathbb{R}^{4}$.

Lemma 1. The orientation on $\Lambda_{ \pm}^{2} \mathbb{R}^{4}$ determined by the basis $s_{1}^{ \pm}, s_{2}^{ \pm}, s_{3}^{ \pm}$defined by means of an oriented orthonormal basis $\left\{e_{1}, \ldots, e_{4}\right\}$ of $\mathbb{R}^{4}$ does not depend on the choice of $\left\{e_{1}, \ldots, e_{4}\right\}$.

Proof. Let $\left\{s_{i}^{\prime}=s_{i}^{\prime+}\right\}$ and $\left\{s_{i}=s_{i}^{+}\right\}$be the bases of $\Lambda_{+}^{2} \mathbb{R}^{4}$ defined by means of two oriented orthonormal bases $\left\{e_{1}^{\prime}, \ldots, e_{4}^{\prime}\right\}$ and $\left\{e_{1}, \ldots, e_{4}\right\}$ of $\mathbb{R}^{4}$. Denote by $A \in S O(4)$ the transition matrix between these bases. Thanks to L. van Elfrikhof (1897), it is well-known that every matrix $A$ in $S O(4)$ can be represented as the product $A=A_{1} A_{2}$ of two $S O(4)$-matrices of the following types

$$
A_{1}=\left(\begin{array}{cccc}
a & -b & -c & -d \\
b & a & -d & c \\
c & d & a & -b \\
d & -c & b & a
\end{array}\right), \quad A_{2}=\left(\begin{array}{cccc}
p & -q & -r & -s \\
q & p & s & -r \\
r & -s & p & q \\
s & r & -q & p
\end{array}\right),
$$

where $a, \ldots, d, p, \ldots, s$ are real numbers with $a^{2}+b^{2}+c^{2}+d^{2}=1, p^{2}+q^{2}+r^{2}+s^{2}=1$ (isoclinic representation). For an endomorphism $L$ of $\mathbb{R}^{4}$, denote by $\Lambda_{L}$ the induced endomorphism on $\Lambda^{2} \mathbb{R}^{4}$ defined by $\Lambda_{L}(X \wedge Y)=L(X) \wedge L(Y)$. Denote again by $A$ the isomorphism of $\mathbb{R}^{4}$ with matrix $A$ with respect to the basis $e_{1}, \ldots, e_{4}$. Then $s_{i}^{\prime}=\Lambda_{A}\left(s_{i}\right)=\Lambda_{A_{1}} \circ \Lambda_{A_{2}}\left(s_{i}\right), i=1,2,3$. One easily computes that $\Lambda_{A_{2}}\left(s_{i}\right)=s_{i}$, and that $\Lambda_{A_{1}}\left(s_{i}\right)$ is a basis of $\Lambda_{+}^{2} \mathbb{R}^{4}$ whose transition matrix is

$$
\left(\begin{array}{ccc}
a^{2}+b^{2}-\left(c^{2}+d^{2}\right) & -2(a d-b c) & 2(a c+b d) \\
2(a d+b c) & a^{2}+c^{2}-\left(b^{2}+d^{2}\right) & -2(a b-c d) \\
-2(a c-b d) & 2(a b+c d) & a^{2}+d^{2}-\left(b^{2}+c^{2}\right)
\end{array}\right) .
$$

The determinant of the latter matrix is $\left(a^{2}+b^{2}+c^{2}+d^{2}\right)^{3}=1$. This proves the statement for $\Lambda_{+}^{2} \mathbb{R}^{4}$. Changing the orientation of $\mathbb{R}^{4}$ interchanges the roles of $\Lambda_{+}^{2} \mathbb{R}^{4}$ and $\Lambda_{-}^{2} \mathbb{R}^{4}$. Therefore, the statement holds for $\Lambda_{-}^{2} \mathbb{R}^{4}$ as well.

The orientation of $\Lambda_{ \pm}^{2}$ induced by a basis $\left\{s_{1}^{ \pm}, s_{2}^{ \pm}, s_{3}^{ \pm}\right\}$will be called "canonical".

Remark. The map assigning the coset of the matrix above in $S O(3) / S O(2)=S^{2}$ to the unit quaternion $q=a+i b+j c+k d$ is the Hopf map $S^{3} \rightarrow S^{2}$.

Consider the 3 -dimensional Euclidean space $\left(\Lambda_{ \pm}^{2} \mathbb{R}^{4}, g\right)$ with its canonical orientation and denote by $\times$ the usual vector-cross product on it. Then, if $a, b \in \Lambda_{ \pm}^{2} \mathbb{R}^{4}$, the isomorphism $\Lambda^{2} \mathbb{R}^{4} \cong s o(4)$ sends $a \times b$ to $\pm \frac{1}{2}\left[K_{a}, K_{b}\right]$. Thus, if $J \in F_{ \pm}\left(\mathbb{R}^{4}\right)$ and $Q \in T_{J} F\left(\mathbb{R}^{4}\right)=T_{J} F_{ \pm}\left(\mathbb{R}^{4}\right)$, we have

$$
(\mathcal{J} Q)^{\wedge}= \pm\left(J^{\wedge} \times Q^{\wedge}\right) .
$$

Now let $(M, g)$ be an oriented Riemannian manifold of dimension four.

According to the considerations above, the twistor space of such a manifold has two connected components, which can be identified with the unit sphere subbundles $\mathcal{Z}_{ \pm}$of the bundles $\Lambda_{ \pm}^{2} T M \rightarrow M$, the eigensubbundles of the bundle $\pi: \Lambda^{2} T M \rightarrow M$ corresponding to the eigenvalues \pm 1 of the Hodge star operator. These are the positive and the negarive twistor spaces of $(M, g)$. If $\sigma \in \mathcal{Z}_{ \pm}$, then $K_{\sigma}$ is a complex structure on the vector space $T_{\pi(\sigma)} M$ compatible with the metric $g$ and \pm the orientation of $M$. Note that, since $g\left(K_{\sigma} X, Y\right)=2 g(\sigma, X \wedge Y)$, the 2 -vector $2 \sigma$ is dual to the fundamental 2-form of $\left(g, K_{\sigma}\right)$. 
The Levi-Civita connection $\nabla$ of $M$ preserves the bundles $\Lambda_{ \pm}^{2} T M$, so it induces a metric connection on each of them denoted again by $\nabla$. The horizontal distribution of $\Lambda_{ \pm}^{2} T M$ with respect to $\nabla$ is tangent to the twistor space $\mathcal{Z}_{ \pm}$.

The manifold $\mathcal{Z}_{ \pm}$admits two almost complex structures $\mathcal{J}_{1}$ and $\mathcal{J}_{2}$ compatible with each metric $h_{t}$ defined, respectively, by Atiyah-Hitchin-Singer [2], and EellsSalamon [16. On a vertical space $\mathcal{V}_{J}, \mathcal{J}_{1}$ is defined to be the complex structure $\mathcal{J}_{J}$ of the fibre through $J$, while $\mathcal{J}_{2}$ is defined as the conjugate complex structure, i.e. $\mathcal{J}_{2} \mid \mathcal{V}_{J}=-\mathcal{J}_{J}$. On a horizontal space $\mathcal{H}_{J}, \mathcal{J}_{1}$ and $\mathcal{J}_{2}$ are both defined to be the lift to $\mathcal{H}_{J}$ of the endomorphism $J$ of $T_{\pi(J)} M$. Thus, if $\sigma \in \mathcal{Z}_{ \pm}$

$$
\begin{gathered}
\mathcal{J}_{k}\left|\mathcal{H}_{\sigma}=\left(\pi_{*} \mid \mathcal{H}_{\sigma}\right)^{-1} \circ K_{\sigma} \circ \pi_{*}\right| \mathcal{H}_{\sigma} . \\
\mathcal{J}_{k} V=(-1)^{k} \sigma \times V \text { for } V \in \mathcal{V}_{\sigma}, \quad k=1,2 .
\end{gathered}
$$

Let $R$ be the curvature tensor of the Levi-Civita connection of $(M, g)$; we adopt the following definition for the curvature tensor $R: R(X, Y)=\nabla_{[X, Y]}-\left[\nabla_{X}, \nabla_{Y}\right]$. Then the curvature operator $\mathcal{R}$ is the self-adjoint endomorphism of $\Lambda^{2} T M$ defined by

$$
g(\mathcal{R}(X \wedge Y), Z \wedge T)=g(R(X, Y) Z, T), \quad X, Y, Z, T \in T M .
$$

The curvature tensor of the connection on the bundle $\Lambda^{2} T M$ induced by the LeviCivita connection on $T M$ will also be denoted by $R$.

The following easily verified formulas are useful in various computations on $\mathcal{Z}_{ \pm}$.

$$
g(R(a) b, c)= \pm g(\mathcal{R}(a), b \times c))
$$

for $a \in \Lambda^{2} T_{p} M, b, c \in \Lambda_{ \pm}^{2} T_{p} M$,

$$
\begin{gathered}
K_{b} \circ K_{c}=-g(b, c) I d \pm K_{b \times c}, \quad b, c \in \Lambda_{ \pm}^{2} T_{p} M . \\
g\left(\sigma \times V, X \wedge K_{\sigma} Y\right)=g\left(\sigma \times V, K_{\sigma} X \wedge Y\right)= \pm g(V, X \wedge Y)
\end{gathered}
$$

for $\sigma \in \mathcal{Z}_{ \pm}, V \in \mathcal{V}_{\sigma}, X, Y \in T_{\pi(\sigma)} M$.

Denote by $\mathcal{B}: \Lambda^{2} T M \rightarrow \Lambda^{2} T M$ the endomorphism corresponding to the traceless Ricci tensor. If $s$ denotes the scalar curvature of $(M, g)$ and $\rho: T M \rightarrow T M$ is the Ricci operator, $g(\rho(X), Y)=\operatorname{Ricci}(X, Y)$, we have

$$
\mathcal{B}(X \wedge Y)=\rho(X) \wedge Y+X \wedge \rho(Y)-\frac{s}{2} X \wedge Y .
$$

Note that $\mathcal{B}$ sends $\Lambda_{ \pm}^{2} T M$ into $\Lambda_{\mp}^{2} T M$. Let $\mathcal{W}: \Lambda^{2} T M \rightarrow \Lambda^{2} T M$ be the endomorphism corresponding to the Weyl conformal tensor. Denote the restriction of $\mathcal{W}$ to $\Lambda_{ \pm}^{2} T M$ by $\mathcal{W}_{ \pm}$, so $\mathcal{W}_{ \pm}$sends $\Lambda_{ \pm}^{2} T M$ to $\Lambda_{ \pm}^{2} T M$ and vanishes on $\Lambda_{\mp}^{2} T M$.

It is well known that the curvature operator decomposes as (31, see e.g. [3, Chapter $1 \mathrm{H}])$

$$
\mathcal{R}=\frac{s}{6} I d+\mathcal{B}+\mathcal{W}_{+}+\mathcal{W}_{-}
$$

Note that this differ by a factor $1 / 2$ from [3] because of the factor $1 / 2$ in our definition of the induced metric on $\Lambda^{2} T M$.

The Riemannian manifold $(M, g)$ is Einstein exactly when $\mathcal{B}=0$. It is called self-dual (anti-self-dual), if $\mathcal{W}_{-}=0$ (resp. $\left.\mathcal{W}_{+}=0\right)$.

It is a well-known result by Atiyah-Hitchin-Singer [2] that the almost complex structure $\mathcal{J}_{1}$ on $\mathcal{Z}_{-}$(resp. $\mathcal{Z}_{+}$) is integrable (i.e. comes from a complex structure) if and only if $(M, g)$ is self-dual (resp. anti-self-dual). On the other hand the 
almost complex structure $\mathcal{J}_{2}$ is never integrable by a result of Eells-Salamon [16, but nevertheless it plays a useful role in harmonic map theory.

\section{ThE STANDARD VARIATION WITH COMPACT SUPPORT OF AN ALMOST HERMITIAN STRUCTURE THROUGH SECTIONS OF THE TWISTOR SPACE}

Now suppose that $(N, h)$ is a Riemannian manifold, which admits an almost Hermitian structure $J$, i.e. a section of the bundle $\pi: \mathcal{T} \rightarrow N$. Take a section $V$ with compact support $K$ of the bundle $J^{*} \mathcal{V} \rightarrow N$, the pull-back under $J$ of the vertical bundle $\mathcal{V} \rightarrow \mathcal{T}$. There exists an $\varepsilon>0$ such that, for every point $I$ of the compact set $J(K)$, the exponential map $\exp _{I}$ is a diffeomorphism of the $\varepsilon$-ball in $T_{I} \mathcal{T}$. The function $\|V\|_{h_{1}}$ is bounded on $N$, so there exists a number $\varepsilon^{\prime}>0$ such that $\|s V(p)\|_{h_{1}}<\varepsilon$ for every $p \in N$ and $s \in\left(-\varepsilon^{\prime}, \varepsilon^{\prime}\right)$. Set $J_{s}(p)=\exp _{J(p)}[s V(p)]$ for $p \in N$ and $s \in\left(-\varepsilon^{\prime}, \varepsilon^{\prime}\right)$. For every fixed $p \in N$, the curve $s \rightarrow \exp _{J(p)}[s V(p)]$ is a geodesic with initial velocity vector $V(p)$ which is tangent to the fibre $\mathcal{T}_{p}$ of $\mathcal{T}$ through $J(p)$. Since this fibre is a totally geodesic submanifold, the whole curve lies in it. Hence $J_{s}$ is a section of $\mathcal{T}$, i.e. an almost Hermitian structure on $(N, h)$, such that $J_{s}=J$ on $N \backslash K$.

In particular, this shows that if $(N, h)$ admits a compatible almost complex structure $J$, then it possesses many such structures.

\section{The Energy functional On SECtions of The TWistor SPACE}

If $D$ is a relatively compact open subset of a Riemannian manifold $(N, h)$, the energy functional assigns to every compatible almost complex structure $J$ on $(N, h)$, considered as a map $J:(N, h) \rightarrow\left(\mathcal{T}, h_{t}\right)$, the integral

$$
E_{\Omega}(J)=\int_{D}\left\|J_{*}\right\|_{h, h_{t}}^{2} \text { vol }
$$

where the norm is taken with respect to $h$ and $h_{t}$.

A compatible almost complex structure $J$ is said to be a harmonic section ("a harmonic almost complex structure" in the terminology of [36]), if for every $D$ it is a critical point of the energy functional under variations of $J$ through sections of the twistor space of $(N, h)$.

We have $J_{*} X=\nabla_{X} J+X^{h}$ for every $X \in T N$ where $X^{h}$ is the horizontal lift of $X$ (and $\nabla_{X} J$ is the vertical part of $J_{*} X$ ). Therefore $\left\|J_{*}\right\|^{2}=t\|\nabla J\|_{h}^{2}+$ $(\operatorname{dim} N) \operatorname{vol}(D)$. It follows that the critical points of the energy functional $E_{\Omega}$ coincide with the critical points of the vertical energy functional

$$
J \rightarrow \int_{D}\|\nabla J\|^{2} v o l
$$

and do not depend on the particular choice of the parameter $t$. Another obvious consequence is that the Kähler structures provide the absolute minimum of the energy functional.

The Euler-Lagrange equation for the critical points of the energy functional under variations through sections of the twistor bundle has been obtained by $\mathrm{C}$. Wood.

Theorem 1. (35, 36]) A compatible almost complex structure $J$ is a harmonic section if and only if

$$
\left[J, \nabla^{*} \nabla J\right]=0
$$


where $\nabla^{*}$ is the formal adjoint operator of $\nabla$.

Remark. Suppose that $N$ is oriented and $J$ is an almost Hermitian structure on $(N, h)$ yielding the orientation of $N$, so it is a section of the positive twistor bundle $\mathcal{T}_{+}$. Every variation of $J$ with compact support consisting of sections of the total twistor space $\mathcal{T}$ contains a subvariation consisting of sections of $\mathcal{T}_{+}$. Thus $J$ is a critical point of the energy functional under variations with compact support through sections of the total twistor space $\mathcal{T}$, if and only if it is a critical point under variations through sections of $\mathcal{T}_{+}$.

Examples of non-Kähler almost Hermitian structures, which are harmonic sections.

1. (35) The standard nearly Kähler structure on $S^{6}$.

2. (35]) The Calabi-Eckmann complex structure on $S^{2 p+1} \times S^{2 q+1}$ with the product metric.

3. (35]) The Abbena-Thurston [1, 33, almost Kähler structure on (the real Heisenberg group $\left.\times S^{1}\right) /($ discrete subgroup).

4. The complex structure of the Iwasawa manifold - (the complex Heisenberg group)/(discrete subgroup).

G. Bor, L. Hernández-Lamoneda and M. Salvai 4 have given sufficient conditions for an almost Hermitian complex structure to minimize the energy functional among sections of the twistor bundle.

Theorem 2. (4) Let $(N, h)$ be a compact Riemannian manifold and let $J$ be a compatible almost complex structure on it. Suppose that

(1) $\operatorname{dim} N=4$, the manifold $(N, h)$ is anti-self-dual and the almost Hermitian structure $(h, J)$ is Hermitian, or almost Kähler,

or

(2) $\operatorname{dim} N \geq 6,(N, h)$ is conformally flat and the almost Hermitian structure $(h, J)$ is of Gray-Hervella [19] type $W_{1} \oplus W_{4}$.

Then the almost complex structure $J$ is an energy minimizer.

Examples of non-Kähler minimizers of the energy functional.

1. (4) C. LeBrun 27 has constructed anti-self-dual Hermitian structures on the blow-ups $\left(S^{3} \times S^{1}\right) \sharp n \overline{\mathbb{C P}^{2}}$ of the Hopf surface $S^{3} \times S^{1}$. Blow-ups do not affect the first Betti number, so any blow up of the Hopf surface has Betti number one, and hence it does not admit a Kähler metric.

2. I. Kim [24 has shown the existence of anti-self-dual strictly almost Kähler structures on $\mathbb{C P}^{2} \sharp n \overline{\mathbb{C P}^{2}}, n \geq 11,\left(S^{2} \times \Sigma\right) \sharp n \overline{\mathbb{C P}^{2}}$, genus $\Sigma \geq 2,\left(S^{2} \times T^{2}\right) \sharp n \overline{\mathbb{C P}^{2}}$, $n \geq 6$.

3. (4) The standard Hermitian structure on the Hopf manifold $S^{2 p+1} \times S^{1}$ is conformally flat and locally conformally Kähler, and hence of Grey-Hervella class $W_{4}$.

Let $(N, h, J)$ be an almost Hermitian manifold and $\Omega(X, Y)=h(J X, Y)$ its fundamental 2 -form. Then the Euler-Lagrange equation $\left[J, \nabla^{*} \nabla J\right]=0$ is equivalent to the identity

$$
\left(\nabla^{*} \nabla \Omega\right)(X, Y)=\left(\nabla^{*} \nabla \Omega\right)(J X, J Y), \quad X, Y \in T N .
$$

Note that for the rough Laplacian $\nabla^{*} \nabla$ we have $\nabla^{*} \nabla \Omega=-$ Trace $\nabla^{2} \Omega$.

The following simple observation is useful in many cases. Let $\widehat{\Omega}$ be the section of $\Lambda^{2} T N$ corresponding to the the 2 -form $\Omega$ under the isomorphism $\Lambda^{2} T N \cong \Lambda^{2} T^{*} N$ 
determined by the metric $g$ on $\Lambda^{2} T N$ defined by means of the metric $h$ on $T N$ via (11). Thus, $h(\widehat{\Omega}, X \wedge Y)=\Omega(X, Y)$, and if $E_{1}, \ldots, E_{m}, J E_{1}, \ldots, J E_{m}$ is an orthonormal frame of $T N$, then

$$
\widehat{\Omega}=2 \sum_{k=1}^{m} E_{k} \wedge J E_{k} .
$$

Denote by $\mathcal{R}(\Omega)$ the 2 -form corresponding to $\mathcal{R}(\widehat{\Omega})$; we have

$$
\mathcal{R}(\Omega)(X, Y)=h(\mathcal{R}(\widehat{\Omega}), X \wedge Y) .
$$

Lemma 2. (9]) A compatible almost complex structure $J$ on a Riemannian manifold $(N, h)$ is a harmonic section if and only if

$$
\Delta \Omega(X, Y)-\Delta \Omega(J X, J Y)=\mathcal{R}(\Omega)(X, Y)-\mathcal{R}(\Omega)(J X, J Y), \quad X, Y \in T N,
$$

where $\Delta$ is the Laplace-de Rham operator of $(N, h)$.

Proof. By the Weitzenböck formula

$$
\begin{gathered}
\Delta \Omega(X, Y)-\left(\nabla^{*} \nabla \Omega\right)(X, Y) \\
=\operatorname{Trace}\{Z \rightarrow(R(Z, Y) \Omega)(Z, X)-(R(Z, X) \Omega)(Z, Y)\},
\end{gathered}
$$

$X, Y \in T N$ (see, for example, [15]). We have

$$
\begin{gathered}
(R(Z, Y) \Omega)(Z, X)=-\Omega(R(Z, Y) Z, X)-\Omega(Z, R(Z, Y) X) \\
=h(R(Z, Y) Z, J X)+h(R(Z, Y) X, J Z) .
\end{gathered}
$$

Hence

$$
\begin{gathered}
\Delta \Omega(X, Y)-\left(\nabla^{*} \nabla \Omega\right)(X, Y) \\
=\operatorname{Ricci}(Y, J X)-\operatorname{Ricci}(X, J Y) \\
+\operatorname{Trace}\{Z \rightarrow h(R(Z, Y) X, J Z-h(R(Z, X) Y, J Z)\}
\end{gathered}
$$

By the algebraic Bianchi identity

$$
\begin{gathered}
h(R(Z, Y) X, J Z)-h(R(Z, X) Y, J Z)=h(R(X, Y) Z, J Z) \\
=h(\mathcal{R}(Z \wedge J Z), X \wedge Y) .
\end{gathered}
$$

We have

$$
\begin{gathered}
\operatorname{Trace}\{Z \rightarrow h(\mathcal{R}(Z \wedge J Z), X \wedge Y)\}=2 \sum_{k=1}^{m} h\left(\mathcal{R}\left(E_{k} \wedge J E_{k}\right), X \wedge Y\right) \\
=h(\mathcal{R}(\widehat{\Omega}), X \wedge Y)=\mathcal{R}(\Omega)(X, Y) .
\end{gathered}
$$

Thus

$$
\Delta \Omega(X, Y)-\left(\nabla^{*} \nabla \Omega\right)(X, Y)=\operatorname{Ricci}(Y, J X)-\operatorname{Ricci}(X, J Y)+\mathcal{R}(\Omega)(X, Y),
$$

and the result follows from (9).

\section{The Atiyah-Hitchin-Singer and Eells-Salamon almost complex STRUCTURES AS HARMONIC SECTIONS}

Lemma 2 has been used to prove the following statement. 
Theorem 3. (9]) Let $(M, g)$ be an oriented Riemannian 4-manifold and let $\left(\mathcal{Z}, h_{t}\right)$ be its negative twistor space. Then:

(i) The Atiyah-Hitchin-Singer almost-complex structure $\mathcal{J}_{1}$ on $\left(\mathcal{Z}, h_{t}\right)$ is a harmonic section if and only if $(M, g)$ is a self-dual manifold.

(ii) The Eells-Salamon almost-complex structure $\mathcal{J}_{2}$ on $\left(\mathcal{Z}, h_{t}\right)$ is a harmonic section if and only if $(M, g)$ is a self-dual manifold with constant scalar curvature.

In order to apply Lemma 2, one needs to compute the Laplacian of the fundamental 2-form $\Omega_{k, t}(A, B)=h_{t}\left(\mathcal{J}_{k} A, B\right), k=1$, 2, of the almost-Hermitian structure $\left(h_{t}, \mathcal{J}_{k}\right)$ on $\mathcal{Z}$. A computation, involving coordinate-free formulas for the differential and codifferential of $\Omega_{k, t}$ (29]), gives the following expression for the Laplacian of $\Omega_{k, t}$ in terms of the base manifold $(M, g)$.

Lemma 3. (9]) Let $V$ be a vertical vector of $\mathcal{Z}$ at a point $\sigma$ and $X, Y \in T_{\pi(\sigma)} M$. Then

$$
\Delta \Omega_{k, t}\left(X^{h}, Y^{h}\right)_{\sigma}=g\left(\frac{4 \sigma}{t}+2(-1)^{k} \mathcal{R}(\sigma), X \wedge Y\right)+\operatorname{tg}(R(X \wedge Y) \sigma, R(\sigma) \sigma)
$$

and

$$
\Delta \Omega_{k, t}\left(V, X^{h}\right)_{\sigma}=(-1)^{k+1} \operatorname{tg}(\delta \mathcal{R}(X), V)-\operatorname{tg}\left(\left(\nabla_{X} \mathcal{R}\right)(\sigma), \sigma \times V\right) .
$$

To compute the curvature terms $\mathcal{R}_{\mathcal{Z}}\left(\Omega_{k, t}\right)$ in (10) one can use the following coordinate-free formula for the curvature of the twistor space.

Proposition 1. ([8]) Let $\mathcal{Z}$ be the negative twistor space of an oriented Riemannian 4-manifold $(M, g)$ with curvature tensor $R$. Let $E, F \in T_{\sigma} \mathcal{Z}$ and $X=\pi_{*} E, Y=$ $\pi_{*} F, V=\mathcal{V} E, W=\mathcal{V} F$ where $\mathcal{V}$ means "the vertical part". Then

$$
\begin{gathered}
h_{t}\left(R_{\mathcal{Z}}(E, F) E, F\right)=g(R(X, Y) X, Y) \\
-t g\left(\left(\nabla_{X} \mathcal{R}\right)(X \wedge Y), \sigma \times W\right)+\operatorname{tg}\left(\left(\nabla_{Y} \mathcal{R}\right)(X \wedge Y), \sigma \times V\right) \\
-3 \operatorname{tg}(\mathcal{R}(\sigma), X \wedge Y) g(\sigma \times V, W) \\
-t^{2} g(R(\sigma \times V) X, R(\sigma \times W) Y)+\frac{t^{2}}{4}\|R(\sigma \times W) X+R(\sigma \times V) Y\|^{2} \\
-\frac{3 t}{4}\|R(X, Y) \sigma\|^{2}+t\left(\|V\|^{2}\|W\|^{2}-g(V, W)^{2}\right),
\end{gathered}
$$

where the norm of the vertical vectors is taken with respect to the metric $g$ on $\Lambda_{-}^{2} T M$.

Using this formula, the well-known expression of the Levi-Civita curvature tensor by means of sectional curvatures (cf. e.g. [20, $\S 3.6$, p. 93, formula (15)]), and the differential Bianchi identity one gets the following.

Corollary 1. Let $\sigma \in \mathcal{Z}, X, Y, Z, T \in T_{\pi(\sigma)} M$, and $U, V, W, W^{\prime} \in \mathcal{V}_{\sigma}$. Then

$$
\begin{gathered}
h_{t}\left(R_{\mathcal{Z}}\left(X^{h}, Y^{h}\right) Z^{h}, T^{h}\right)_{\sigma}=g(R(X, Y) Z, T) \\
-\frac{3 t}{12}[2 g(R(X, Y) \sigma, R(Z, T) \sigma)-g(R(X, T) \sigma, R(Y, Z) \sigma) \\
+g(R(X, Z) \sigma, R(Y, T) \sigma)] .
\end{gathered}
$$




$$
\begin{gathered}
h_{t}\left(R_{\mathcal{Z}}\left(X^{h}, Y^{h}\right) Z^{h}, U\right)_{\sigma}=-\frac{t}{2} g\left(\nabla_{Z} \mathcal{R}(X \wedge Y), \sigma \times U\right) . \\
h_{t}\left(R_{\mathcal{Z}}\left(X^{h}, U\right) Y^{h}, V\right)_{\sigma}=\frac{t^{2}}{4} g(R(\sigma \times V) X, R(\sigma \times U) Y) \\
+\frac{t}{2} g(\mathcal{R}(\sigma), X \wedge Y) g(\sigma \times V, U) . \\
h_{t}\left(R_{\mathcal{Z}}\left(X^{h}, Y^{h}\right) U, V\right)_{\sigma}=\frac{t^{2}}{4}[g(R(\sigma \times V) X, R(\sigma \times U) Y) \\
+t g(\mathcal{R}(\sigma), X \wedge Y) g(\sigma \times V, U) \\
h_{t}\left(R_{\mathcal{Z}}\left(X^{h}, U\right) V, W\right)=0, \quad h_{t}\left(R_{\mathcal{Z}}(U, V) W, W^{\prime}\right)=g(U, W) g\left(V, W^{\prime}\right)-g\left(U, W^{\prime}\right) g(V, W) .
\end{gathered}
$$

This implies

Lemma 4. (9]) Let $V, W$ be vertical vectors of $\mathcal{Z}$ at a point $\sigma$ and $X, Y \in T_{\pi(\sigma)} M$. Then

$$
\begin{aligned}
\mathcal{R}_{t}\left(\Omega_{k, t}\right)\left(X^{h}, Y^{h}\right)_{\sigma}= & 2\left[1+(-1)^{k+1}\right] g(\mathcal{R}(\sigma), X \wedge Y)-\operatorname{tg}(R(X \wedge Y) \sigma, R(\sigma) \sigma) \\
& -\frac{t}{2} \operatorname{Trace}\left\{Z \rightarrow g\left(R(X \wedge Z) \sigma, R\left(Y \wedge K_{\sigma} Z\right) \sigma\right)\right\} \\
& -\frac{t}{2}(-1)^{k} \operatorname{Trace}\left\{\mathcal{V}_{\sigma} \ni \tau \rightarrow g(R(\tau) X, R(\sigma \times \tau) Y)\right\},
\end{aligned}
$$

where the latter trace is taken with respect to the metric $g$ on $\mathcal{V}_{\sigma}$,

$$
\mathcal{R}_{t}\left(\Omega_{k, t}\right)\left(V, X^{h}\right)_{\sigma}=\operatorname{tg}\left(\left(\nabla_{X} \mathcal{R}\right)(\sigma), \sigma \times V\right)
$$

and

$$
\begin{aligned}
\mathcal{R}_{t}\left(\Omega_{k, t}\right)(V, W)_{\sigma}= & 2\left[(-1)^{k+1}+\operatorname{tg}(\mathcal{R}(\sigma), \sigma)\right] g(V, \sigma \times W) \\
& +\frac{t^{2}}{2} \operatorname{Trace}\left\{Z \rightarrow g\left(R(\sigma \times V) K_{\sigma} Z, R(\sigma \times W) Z\right)\right\} .
\end{aligned}
$$

Proof of Theorem [3. According to Lemmas 2, 3] and 4, the almost complex structure $\mathcal{J}_{n}$ is a harmonic section if and only if the following two conditions are satisfied:

$$
\begin{gathered}
4 g\left(\mathcal{R}(\sigma), X \wedge Y-K_{\sigma} X \wedge K_{\sigma} Y\right)= \\
t \operatorname{Trace}\left\{Z \rightarrow g\left(R(X \wedge Z) \sigma, R\left(Y \wedge K_{\sigma} Z\right) \sigma\right)-g\left(R\left(K_{\sigma} X \wedge Z\right) \sigma, R\left(K_{\sigma} Y \wedge K_{\sigma} Z\right) \sigma\right)\right\} \\
+t(-1)^{k} \operatorname{Trace}\left\{\mathcal{V}_{\sigma} \ni \tau \rightarrow g(R(\tau) X, R(\sigma \times \tau) Y)-g\left(R(\tau) K_{\sigma} X, R(\sigma \times \tau) K_{\sigma} Y\right)\right\}
\end{gathered}
$$

and

$$
g\left(\delta \mathcal{R}\left(K_{\sigma} X\right), \sigma \times V\right)=(-1)^{k} g(\delta \mathcal{R}(X), V)
$$

for every $\sigma \in \mathcal{Z}, V \in \mathcal{V}_{\sigma}$ and $X, Y \in T_{\pi(\sigma)} M$.

We shall show that condition (15) is equivalent to $(M, g)$ being a self-dual manifold. Note first that (15) holds for every $X, Y \in T_{\pi(\sigma)} M$ if and only if it holds for every $X, Y \in T_{\pi(\sigma)} M$ with $\|X\|=\|Y\|=1$ and $X \perp Y, K_{\sigma} Y$. For every such $X, Y$ there is a unique $\tau \in \mathcal{V}_{\sigma},\|\tau\|=1$, such that $Y=K_{\tau} X$, namely 
$\tau=X \wedge Y-K_{\sigma} X \wedge K_{\sigma} Y$; conversely, if $\tau \in \mathcal{V}_{\sigma},\|\tau\|=1$ and $Y=K_{\tau} X$, then $X \perp Y, K_{\sigma} Y$ in view of (6). Thus, (15) holds if and only if it holds for every $X \in T_{\pi(\sigma)} M$ and $Y=K_{\tau} X$ with $\|X\|=1, \tau \in \mathcal{V}_{\sigma},\|\tau\|=1$. Given such $X$ and $\tau$, the vectors $E_{1}=X, E_{2}=K_{\sigma} X, E_{3}=K_{\tau} X, E_{4}=K_{\sigma \times \tau} X$ constitute an oriented orthonormal basis of $T_{\pi(\sigma} M$ such that $s_{1}^{-}=\sigma, s_{2}^{-}=\tau, s_{3}^{-}=\sigma \times \tau$, where $s_{1}^{-}, s_{2}^{-}, s_{3}^{-}$are defined by means of $\left\{E_{1}, \ldots, E_{4}\right\}$ via (2). Using the bases $\left\{E_{1}, \ldots, E_{4}\right\}$ of $T_{\pi(\sigma)} M$ and $\tau, \sigma \times \tau$ of $\mathcal{V}_{\sigma}$ to compute the traces in the right-hand side of (15), we see that identity (15) is equivalent to

$$
\begin{gathered}
4 g(\mathcal{R}(\sigma), \tau)=\operatorname{tg}(R(\sigma) \sigma, R(\tau) \sigma) \\
+t(-1)^{k} g(R(\tau) \sigma, R(\sigma \times \tau)(\sigma \times \tau))-t(-1)^{k} g(R(\tau)(\sigma \times \tau), R(\sigma \times \tau) \sigma)
\end{gathered}
$$

for every $\sigma, \tau \in \mathcal{Z}, \pi(\sigma)=\pi(\tau), \sigma \perp \tau$. Using (5) we easily see also that the latter identity is equivalent to

$$
\begin{gathered}
4 g(\mathcal{R}(\sigma), \tau)=\operatorname{tg}(\mathcal{R}(\sigma), \sigma \times \tau) g(\mathcal{R}(\tau), \sigma \times \tau)+\operatorname{tg}(\mathcal{R}(\sigma), \tau) g(\mathcal{R}(\tau), \tau) \\
+t(-1)^{k+1} g(\mathcal{R}(\tau), \sigma \times \tau) g(\mathcal{R}(\sigma \times \tau), \tau)-t(-1)^{k+1} g(\mathcal{R}(\tau), \sigma) g(\mathcal{R}(\sigma \times \tau), \sigma \times \tau) .
\end{gathered}
$$

Writing this identity with $(\sigma, \tau)$ replaced by $(\tau, \sigma)$ and comparing the obtained identity with (17) we get

$$
g(\mathcal{R}(\sigma), \tau)[g(\mathcal{R}(\sigma), \sigma)-g(\mathcal{R}(\tau), \tau)]=0 .
$$

Replacing the pair $(\sigma, \tau)$ by $\left(\frac{3 \sigma+4 \tau}{5}, \frac{4 \sigma-3 \tau}{5}\right)$ in (18) and using again this identity, we obtain

$$
[g(\mathcal{R}(\sigma), \sigma)-g(\mathcal{R}(\tau), \tau)]^{2}=4[g(\mathcal{R}(\sigma), \tau)]^{2},
$$

which, together with (18), gives

$$
g(\mathcal{R}(\sigma), \sigma)=g(\mathcal{R}(\tau), \tau), \quad g(\mathcal{R}(\sigma), \tau)=0 .
$$

Thus

$$
g\left(\mathcal{W}_{-}(\sigma), \sigma\right)=g\left(\mathcal{W}_{-}(\tau), \tau\right) \text { and } g\left(\mathcal{W}_{-}(\sigma), \tau\right)=0
$$

Since Trace $\mathcal{W}_{-}=0$, this implies $\mathcal{W}_{-}=0$.

Conversely, if $\mathcal{W}_{-}=0$ we have $\mathcal{R}(\sigma)=\frac{s}{6} \sigma+\mathcal{B}(\sigma)$ where $\mathcal{B}(\sigma) \in \Lambda_{+}^{2} T M$, so it is obvious that identity (17) is satisfied.

To analyze condition (16) we recall that $\delta \mathcal{R}=2 \delta \mathcal{B}(=-d$ Ricci) (cf. e.g. [3]), so it follows from (8) that

$$
\delta \mathcal{R}(X)=-\frac{1}{3} \operatorname{grad} s \wedge X+2 \delta \mathcal{W}(X), X \in T M .
$$

Suppose $\mathcal{W}_{-}=0$. Since $\delta \mathcal{W}_{+}(X) \in \Lambda_{+}^{2} T M$, we have

$$
g(\delta \mathcal{R}(X), V)=\frac{1}{3} g(X \wedge \operatorname{grad} s, V)
$$

for any $V \in \Lambda_{-}^{2} T M$. The latter formula and (7) imply that condition (16) is equivalent (for self-dual manifolds) to the identity

$$
g(V, X \wedge \operatorname{grad} s)=(-1)^{k+1} g(V, X \wedge \operatorname{grad} s) .
$$

Clearly, this identity is satisfied if $k=1$; for $k=2$ it holds if and only if the scalar curvature $s$ is constant. 


\section{The SECOnd fundamental form of an almost Hermitian structure} AS A MAP INTO THE TWISTOR SPACE

Let $J$ be a compatible almost complex structure on a Riemannian manifold $(N, h)$. Then we have a map $J:(N, h) \rightarrow\left(\mathcal{T}, h_{t}\right)$ between Riemannian manifolds. Let $J^{*} T \mathcal{T} \rightarrow N$ be the pull-back of the bundle $T \mathcal{T} \rightarrow \mathcal{T}$ under the map $J: N \rightarrow$ $\mathcal{T}$. We can consider the differential $J_{*}: T N \rightarrow T \mathcal{T}$ as a section of the bundle $\operatorname{Hom}\left(T N, J^{*} T \mathcal{T}\right) \rightarrow N$. Denote by $\widetilde{D}$ the connection on $J^{*} T \mathcal{T}$ induced by the Levi-Civita connection $D$ on $T \mathcal{T}$. The Levi Civita connection $\nabla$ on $T N$ and the connection $\widetilde{D}$ on $J^{*} T \mathcal{T}$ induce a connection $\widetilde{\nabla}$ on the bundle $\operatorname{Hom}\left(T N, J^{*} T \mathcal{T}\right)$. Recall that the second fundamental form of the map $J$ is, by definition, $\widetilde{\nabla} J_{*}$. The map $J:(N, h) \rightarrow\left(\mathcal{T}, h_{t}\right)$ is harmonic if and only if

$$
\text { Trace }_{h} \widetilde{\nabla} J_{*}=0 \text {. }
$$

Recall also that the map $J:(N, h) \rightarrow\left(\mathcal{T}, h_{t}\right)$ is totally geodesic exactly when $\widetilde{\nabla} J_{*}=0$.

Proposition 2. ([11, 10]) For every $X, Y \in T_{p} N$,

$$
\begin{gathered}
\widetilde{\nabla} J_{*}(X, Y)=\frac{1}{2} \mathcal{V}\left(\nabla_{X Y}^{2} J+\nabla_{Y X}^{2} J\right) \\
-\frac{2 t}{n}\left[\left(R\left(\left(J \circ \nabla_{X} J\right)^{\wedge}\right) Y\right)_{J(p)}^{h}+\left(R\left(\left(J \circ \nabla_{Y} J\right)^{\wedge}\right) X\right)_{J(p)}^{h}\right],
\end{gathered}
$$

where $\mathcal{V}$ means "the vertical component", $n=\operatorname{dim} N$, and $\nabla_{X Y}^{2} J=\nabla_{X} \nabla_{Y} J-$ $\nabla_{\nabla_{X} Y} J$ is the second covariant derivative of $J$.

The computation of the second fundamental form is based on several lemmas.

First, we note that identity (5) can be generalized as follows.

Lemma 5. ([7]) For every $a, b \in A\left(T_{p} N\right)$ and $X, Y \in T_{p} N$, we have

$$
G(R(X, Y) a, b)=\frac{2}{n} h\left(R\left([a, b]^{\wedge}\right) X, Y\right) .
$$

Proof. Let $E_{1}, \ldots, E_{n}$ be an orthonormal basis of $T_{p} N$. Then

$$
[a, b]=\frac{1}{2} \sum_{i, j=1}^{n} h\left([a, b] E_{i}, E_{j}\right) E_{i} \wedge E_{j} .
$$

Therefore

$$
\begin{gathered}
h\left(R\left([a, b]^{\wedge}\right) X, Y\right)=\frac{1}{2} \sum_{i, j=1}^{n} h\left(R(X, Y) E_{i}, E_{j}\right)\left[h\left(a b E_{i}, E_{j}\right)+h\left(a E_{i}, b E_{j}\right)\right] \\
=-\frac{1}{2} \sum_{i=1}^{n} h\left(a\left(R(X, Y) E_{i}\right), b E_{i}\right)+\frac{1}{2} \sum_{k=1}^{n} h\left(R(X, Y) a E_{k}, b E_{k}\right) \\
=\frac{n}{2} G(R(X, Y) a, b) .
\end{gathered}
$$

Lemma $[$ implies

$$
h_{t}(R(X, Y) J, V)=\frac{2 t}{n} h\left(R\left([J, V]^{\wedge}\right) X, Y\right)=\frac{4 t}{n} h\left(R\left((J \circ V)^{\wedge}\right) X, Y\right) .
$$


Lemma 6. ([7, 8]) If $X, Y$ are vector fields on $N$, and $V$ is a vertical vector field on $\mathcal{T}$, then

$$
\begin{gathered}
\left(D_{X^{h}} Y^{h}\right)_{I}=\left(\nabla_{X} Y\right)_{I}^{h}+\frac{1}{2} R_{p}(X \wedge Y) I \\
\left(D_{V} X^{h}\right)_{I}=\mathcal{H}\left(D_{X^{h}} V\right)_{I}=-\frac{2 t}{n}\left(R_{p}\left(\left(I \circ V_{I}\right)^{\wedge}\right) X\right)_{I}^{h},
\end{gathered}
$$

where $I \in \mathcal{T}, p=\pi(I), n=\operatorname{dim} N$, and $\mathcal{H}$ means "the horizontal component".

Proof. Identity (21) follows from the Koszul formula for the Levi-Civita connection and the identity $\left[X^{h}, Y^{h}\right]_{I}=[X, Y]_{I}^{h}+R(X, Y) I$.

Let $W$ be a vertical vector field on $\mathcal{T}$. Then

$$
h_{t}\left(D_{V} X^{h}, W\right)=-h_{t}\left(X^{h}, D_{V} W\right)=0,
$$

since the fibres are totally geodesic submanifolds, so $D_{V} W$ is a vertical vector field. Therefore, $D_{V} X^{h}$ is a horizontal vector field. Moreover, $\left[V, X^{h}\right]$ is a vertical vector field, hence $D_{V} X^{h}=\mathcal{H} D_{X^{h}} V$. Thus

$$
h_{t}\left(D_{V} X^{h}, Y^{h}\right)=h_{t}\left(D_{X^{h}} V, Y^{h}\right)=-h_{t}\left(V, D_{X^{h}} Y^{h}\right) .
$$

Now (22) follows from (21) and (20).

Any (local) section $a$ of the bundle $A(T N)$ determines a (local) vertical vector field $\widetilde{a}$ on $\mathcal{T}$ defined by

$$
\widetilde{a}_{I}=\frac{1}{2}(a(p)+I \circ a(p) \circ I), \quad p=\pi(I) .
$$

The next lemma is "folklore".

Lemma 7. If $I \in \mathcal{T}$ and $X$ is a vector field on a neighbourhood of the point $p=\pi(I)$, then

$$
\left[X^{h}, \widetilde{a}\right]_{I}=\left(\widetilde{\nabla_{X} a}\right)_{I}
$$

Let $I \in \mathcal{T}$ and let $U, V \in \mathcal{V}_{I}$. Take section $a$ and $b$ of $A(T N)$ such that $a(p)=U$, $b(p)=V$ for $p=\pi(I)$. Let $\widetilde{a}$ and $\widetilde{b}$ be the vertical vector fields determined by the sections $a$ and $b$. Taking into account the fact that the fibre of $\mathcal{T}$ through the point $I$ is a totally geodesic submanifold, one easily gets by means of the Koszul formula that

$$
\left(D_{\widetilde{a}} \widetilde{b}\right)_{I}=\frac{1}{4}[U V I+I V U+I(U V I+I V U) I]=0 .
$$

Lemma 8. For every $p \in N$, there exists an $h_{t}$-orthonormal frame of vertical vector fields $\left\{V_{\alpha}: \alpha=1, \ldots, m^{2}-m\right\}, m=\frac{1}{2} \operatorname{dim} N$, in a neighbourhood of the point $J(p)$ such that

(1) $\quad\left(D_{V_{\alpha}} V_{\beta}\right)_{J(p)}=0, \alpha, \beta=1, \ldots, m^{2}-m$.

(2) If $X$ is a vector field near the point $p$, then $\left[X^{h}, V_{\alpha}\right]_{J(p)}=0$.

(3) $\nabla_{X_{p}}\left(V_{\alpha} \circ J\right) \perp \mathcal{V}_{J(p)}$.

Proof. Let $E_{1}, \ldots, E_{n}$ be an orthonormal frame of $T N$ in a neighbourhood of $p$ such that $J\left(E_{2 k-1}\right)_{p}=\left(E_{2 k}\right)_{p}, k=1, \ldots, m$, and $\left.\nabla E_{l}\right|_{p}=0, l=1, \ldots, n$. Define sections $S_{i j}, 1 \leq i, j \leq n$, of $A(T N)$ by the formula

$$
S_{i j} E_{l}=\sqrt{\frac{n}{2}}\left(\delta_{i l} E_{j}-\delta_{l j} E_{i}\right), \quad l=1, \ldots, n
$$


Then $S_{i j}, i<j$, form an orthonormal frame of $A(T N)$ with respect to the metric $G(a, b)=-\frac{1}{n} \operatorname{Trace}(a \circ b) ; a, b \in A(T N)$. Set

$$
\begin{gathered}
A_{r, s}=\frac{1}{\sqrt{2}}\left(S_{2 r-1,2 s-1}-S_{2 r, 2 s}\right), \quad B_{r, s}=\frac{1}{\sqrt{2}}\left(S_{2 r-1,2 s}+S_{2 r, 2 s-1}\right), \\
r=1, \ldots, m-1, s=r+1, \ldots, m .
\end{gathered}
$$

Then $\left\{\left(A_{r, s}\right)_{p},\left(B_{r, s}\right)_{p}\right\}$ is a $G$-orthonormal basis of the vertical space $\mathcal{V}_{J(p)}$. Note also that $\left.\nabla A_{r, s}\right|_{p}=\left.\nabla B_{r, s}\right|_{p}=0$. Let $\widetilde{A}_{r, s}$ and $\widetilde{B}_{r, s}$ be the vertical vector fields on $\mathcal{T}$ determined by the sections $A_{r, s}$ and $B_{r, s}$ of $A(T N)$. These vector fields constitute a frame of the vertical bundle $\mathcal{V}$ in a neighbourhood of the point $J(p)$.

Considering $\widetilde{A}_{r, s} \circ J$ as a section of $A(T N)$, we have

$$
\begin{gathered}
\nabla_{X_{p}}\left(\widetilde{A}_{r, s} \circ J\right)=\frac{1}{2}\left\{\left(\nabla_{X_{p}} J\right) \circ\left(A_{r, s}\right)_{p} \circ J_{p}+J_{p} \circ\left(A_{r, s}\right) \circ\left(\nabla_{X_{p}} J\right)\right\} \\
=\frac{1}{2}\left\{-\nabla_{X_{p}} \circ J_{p} \circ\left(A_{r, s}\right)_{p}+J_{p} \circ\left(A_{r, s}\right) \circ\left(\nabla_{X_{p}} J\right)\right\} \\
=\frac{1}{2}\left[\left(B_{r, s}\right)_{p}, \nabla_{X_{p}} J\right] .
\end{gathered}
$$

For every $I \in \mathcal{T}$, we have the orthogonal decomposition

$$
A\left(T_{\pi(I)} N\right)=\mathcal{V}_{I} \oplus\left\{S \in A\left(T_{\pi(I)} N\right): I S-S I=0\right\} .
$$

The endomorphisms $\left(B_{r, s}\right)_{p}$ and $\nabla_{X_{p}} J$ of $T_{p} N$ belong to $\mathcal{V}_{J(p)}$, so they anticommute with $J(p)$, hence their commutator commutes with $J(p)$. Therefore the commutator $\left[\left(B_{r, s}\right)_{p}, \nabla_{X_{p}} J\right]$ is $G$-orthogonal to the vertical space at $J$. Thus

$$
\nabla_{X_{p}}\left(\widetilde{A}_{r, s} \circ J\right) \perp \mathcal{V}_{J(p)},
$$

and, similarly, $\nabla_{X_{p}}\left(\widetilde{B}_{r, s} \circ J\right) \perp \mathcal{V}_{J(p)}$.

It is convenient to denote the elements of the frame $\left\{\widetilde{A}_{r, s}, \widetilde{B}_{r, s}\right\}$ by $\left\{\widetilde{V}_{1}, \ldots, \widetilde{V}_{m^{2}-m}\right\}$. In this way we have a frame of vertical vector fields near the point $J(p)$ with property (3) of the lemma. Properties (1) and (2) are also satisfied by this frame according to (23) and Lemma 7 respectively. In particular,

$$
\left(\widetilde{V}_{\gamma}\right)_{J(p)}\left(h_{t}\left(\widetilde{V}_{\alpha}, \widetilde{V}_{\beta}\right)\right)=0, \quad \alpha, \beta, \gamma=1, \ldots, m^{2}-m .
$$

Note also that, in view of (22),

$$
\mathcal{V}\left(D_{X^{h}} \widetilde{V}_{\alpha}\right)_{J(p)}=\left[X^{h}, \widetilde{V}_{\alpha}\right]_{J(p)}=0,
$$

hence

$$
X_{J(p)}^{h}\left(h_{t}\left(\widetilde{V}_{\alpha}, \widetilde{V}_{\beta}\right)\right)=0 .
$$

Now it is clear that the $h_{t}$-orthonormal frame $\left\{V_{1}, \ldots, V_{m^{2}-m}\right\}$ obtained from $\left\{\widetilde{V}_{1}, \ldots, \widetilde{V}_{m^{2}-m}\right\}$ by the Gram-Schmidt process has the properties stated in the lemma.

\section{Proof of Proposition 2 .}

Extend the tangent vectors $X$ and $Y$ to vector fields in a neighbourhood of the point $p$. Let $V_{1}, \ldots, V_{m^{2}-m}$ be an $h_{t}$-orthonormal frame of vertical vector fields with properties (1) - (3) stated in Lemma 8 .

We have

$$
J_{*} \circ Y=Y^{h} \circ J+\nabla_{Y} J=Y^{h} \circ J+\sum_{\alpha=1}^{m^{2}-m} h_{t}\left(\nabla_{Y} J, V_{\alpha} \circ J\right)\left(V_{\alpha} \circ J\right),
$$


hence

$$
\begin{aligned}
\widetilde{D}_{X}\left(J_{*} \circ Y\right)= & \left(D_{J_{*} X} Y^{h}\right) \circ J+\sum_{\alpha=1}^{m^{2}-m} h_{t}\left(\nabla_{Y} J, V_{\alpha}\right)\left(D_{J_{*} X} V_{\alpha}\right) \circ J \\
& +t \sum_{\alpha=1}^{m^{2}-m} G\left(\nabla_{X} \nabla_{Y} J, V_{\alpha} \circ J\right)\left(V_{\alpha} \circ J\right) .
\end{aligned}
$$

This, in view of Lemma 6. implies

$$
\begin{gathered}
\widetilde{D}_{X_{p}}\left(J_{*} \circ Y\right)=\left(\nabla_{X} Y\right)_{J(p)}^{h}+\frac{1}{2} R(X \wedge Y) J(p)-\frac{2 t}{n}\left(R\left(\left(J \circ \nabla_{X} J\right)^{\wedge}\right) Y\right)_{J(p)}^{h} \\
+t \sum_{\alpha=1}^{m^{2}-m} G\left(\nabla_{X_{p}} \nabla_{Y} J, V_{\alpha} \circ J\right)_{p} V_{\alpha}(J(p)) \\
-\frac{2 t}{n}\left(R\left(\left(J \circ \nabla_{Y} J\right)^{\wedge}\right) X\right)_{J(p)}^{h} \\
=\left(\nabla_{X_{p}} Y\right)_{J(p)}^{h}+\frac{1}{2} \mathcal{V}\left(\nabla_{X_{p}} \nabla_{Y} J+\nabla_{Y_{p}} \nabla_{X} J\right)+\frac{1}{2} \nabla_{[X, Y]_{p}} J \\
\left.-\frac{2 t}{n}\left[R\left(\left(J \circ \nabla_{X} J\right)^{\wedge}\right) Y\right)_{J(p)}^{h}+\left(R\left(\left(J \circ \nabla_{Y} J\right)^{\wedge}\right) X\right)_{J(p)}^{h}\right] .
\end{gathered}
$$

It follows that

$$
\begin{aligned}
& \widetilde{\nabla} J_{*}(X, Y)=\widetilde{D}_{X_{p}}\left(J_{*} \circ Y\right)-\left(\nabla_{X} Y\right)_{\sigma}^{h}-\nabla_{\nabla_{X_{p}} Y} J \\
= & \frac{1}{2} \mathcal{V}\left(\nabla_{X_{p}} \nabla_{Y} J-\nabla_{\nabla_{X_{p}} Y} J+\nabla_{Y_{p}} \nabla_{X} J-\nabla_{\nabla_{Y_{p}} X} J\right) \\
- & \left.\frac{2 t}{n}\left[R\left(\left(J \circ \nabla_{X} J\right)^{\wedge}\right) Y\right)_{J(p)}^{h}+\left(R\left(\left(J \circ \nabla_{Y} J\right)^{\wedge}\right) X\right)_{J(p)}^{h}\right] .
\end{aligned}
$$

Proposition 2 implies immediately the following.

Corollary 2. If $(N, h, J)$ is Kähler, the map $J:(N, h) \rightarrow\left(\mathcal{T}, h_{t}\right)$ is a totally geodesic isometric imbedding.

Remark. In view of the decomposition (24), the Euler-Lagrange equation $\left[J, \nabla^{*} \nabla J\right]=$ 0 is equivalent to the condition that the vertical part of $\nabla^{*} \nabla J=-$ Trace $\nabla^{2} J$ vanishes. Thus, by Proposition 2, $J$ is a harmonic section if and only if

$$
\mathcal{V} \text { Trace } \widetilde{\nabla} J_{*}=0 \text {. }
$$

This fact, Proposition 2 and Theorem 3 imply

Corollary 3. (i) $\mathcal{V}$ Trace $\widetilde{\nabla} \mathcal{J}_{1 *}=0$ if and only if $(M, g)$ is self-dual.

(ii) $\mathcal{V}$ Trace $\widetilde{\nabla} \mathcal{J}_{2} *=0$ if and only if $(M, g)$ is self-dual and with constant scalar curvature.

\section{The Atiyah-Hitchin-Singer and Eells-Salamon almost complex STRUCTURES AS HARMONIC MAPS}

The main result in this section is the following.

Theorem 4. Each of the Atiyah-Hitchin-Singer and Eells-Salamon almost complex structures on the negative twistor space $\mathcal{Z}$ of an oriented Riemannian four-manifold $(M, g)$ determines a harmonic map if and only if $(M, g)$ is either self-dual and Einstein, or is locally the product of an open interval in $\mathbb{R}$ and a 3-dimensional Riemannian manifold of constant curvature. 
Remarks. 1. Every manifold that is locally the product of an open interval in $\mathbb{R}$ and a 3-dimensional Riemannian manifold of constant curvature $c$ is locally conformally flat with constant scalar curvature $6 c$. It is not Einstein unless $c=0$, i.e. Ricci flat.

2. According to Theorems 3 and 4 , the conditions under which $\mathcal{J}_{1}$ or $\mathcal{J}_{2}$ is a harmonic section or a harmonic map do not depend on the parameter $t$ of the metric $h_{t}$. Taking certain special values of $t$, we can obtain a metric $h_{t}$ with nice properties (cf., for example, [8, 12, 29]).

The proof is based on several technical lemmas.

Note first that the almost complex structure $\mathcal{J}_{k}, k=1$ or 2 , is a harmonic map if and only if $\mathcal{V}$ Trace $\widetilde{\nabla} \mathcal{J}_{k *}=0$ and $\mathcal{H}$ Trace $\widetilde{\nabla} \mathcal{J}_{k *}=0$. According to Proposition 2 $\mathcal{H}$ Trace $\widetilde{\nabla} \mathcal{J}_{k *}=0, k=1,2$, if and only if for every $\sigma \in \mathcal{Z}$ and every $F \in T_{\sigma} \mathcal{Z}$

$$
\text { Trace } \left._{h_{t}}\left\{T_{\sigma} \mathcal{Z} \ni A \rightarrow h_{t}\left(R_{\mathcal{Z}}\left(\left(\mathcal{J}_{k} \circ D_{A} \mathcal{J}_{k}\right)^{\wedge}\right) A\right), F\right)\right\}=0 .
$$

Set for brevity

$$
\left.\operatorname{Tr}_{k}(F)=\operatorname{Trace}_{h_{t}}\left\{T_{\sigma} \mathcal{Z} \ni A \rightarrow h_{t}\left(R_{\mathcal{Z}}\left(\left(\mathcal{J}_{k} \circ D_{A} \mathcal{J}_{k}\right)^{\wedge}\right) A\right), F\right)\right\} .
$$

Let $\Omega_{k, t}(A, B)=h_{t}\left(\mathcal{J}_{k} A, B\right)$ be the fundamental 2-form of the almost Hermitian manifold $\left(\mathcal{Z}, h_{t}, \mathcal{J}_{k}\right), k=1,2$. Then, for $A, B, C \in T_{\sigma} \mathcal{Z}$,

$$
\left.h_{t}\left(\mathcal{J}_{k} \circ D_{A} \mathcal{J}_{k}\right)^{\wedge}, B \wedge C\right)=-\frac{1}{2} h_{t}\left(\left(D_{A} \mathcal{J}_{k}\right)(B), \mathcal{J}_{k} C\right)=-\frac{1}{2}\left(D_{A} \Omega_{k, t}\right)\left(B, \mathcal{J}_{k} C\right) .
$$

Lemma 9. ([29]) Let $\sigma \in \mathcal{Z}$ and $X, Y \in T_{\pi(\sigma)} M, V \in \mathcal{V}_{\sigma}$. Then

$$
\begin{gathered}
\left(D_{X_{\sigma}^{h}} \Omega_{k, t}\right)\left(Y_{\sigma}^{h}, V\right)=\frac{t}{2}\left[(-1)^{k} g(\mathcal{R}(V), X \wedge Y)-g\left(\mathcal{R}(\sigma \times V), X \wedge K_{\sigma} Y\right)\right], \\
\left(D_{V} \Omega_{k, t}\right)\left(X_{\sigma}^{h}, Y_{\sigma}^{h}\right)=\frac{t}{2} g\left(\mathcal{R}(\sigma \times V), X \wedge K_{\sigma} Y+K_{\sigma} X \wedge Y\right)+2 g(V, X \wedge Y) .
\end{gathered}
$$

Moreover, $\left(D_{A} \Omega_{k, t}\right)(B, C)=0$ when $A, B, C$ are three horizontal vectors at $\sigma$ or at least two of them are vertical.

Corollary 4. Let $\sigma \in \mathcal{Z}, X \in T_{\pi(\sigma)} M, U \in \mathcal{V}_{\sigma}$. If $E_{1}, \ldots, E_{4}$ is an orthonormal basis of $T_{\pi(\sigma)} M$ and $V_{1}, V_{2}$ is an $h_{t}$-orthonormal basis of $\mathcal{V}_{\sigma}$,

$$
\begin{aligned}
&\left(\mathcal{J}_{k} \circ D_{X_{\sigma}} \mathcal{J}_{k}\right)^{\wedge}=-\frac{1}{2} \sum_{i=1}^{4} \sum_{l=1}^{2}\left[g\left(\mathcal{R}\left(\sigma \times V_{l}\right), X \wedge E_{i}\right)\right. \\
&\left.+(-1)^{k} g\left(\mathcal{R}\left(V_{l}\right), X \wedge K_{\sigma} E_{i}\right)\right]\left(E_{i}^{h}\right)_{\sigma} \wedge V_{l}, \\
&\left(\mathcal{J}_{k} \circ D_{U} \mathcal{J}_{k}\right)^{\wedge}=\sum_{1 \leq i<j \leq 4}\left[\frac{t}{2} g\left(\mathcal{R}(\sigma \times U), E_{i} \wedge E_{j}-K_{\sigma} E_{i} \wedge K_{\sigma} E_{j}\right)\right. \\
&\left.-2 g\left(U, E_{i} \wedge K_{\sigma} E_{j}\right)\right]\left(E_{i}^{h}\right)_{\sigma} \wedge\left(E_{j}^{h}\right)_{\sigma} .
\end{aligned}
$$

By Corollary [3] if the vertical part of Trace $\widetilde{\nabla} \mathcal{J}_{k *}$ vanishes, then the manifold $(M, g)$ is self dual. In the case when the base manifold is self-dual, simple, but long computations involving Corollary 4, the algebraic Bianchi identity, Corollary 1, and formula (7), give the next two lemmas, which play an essential role in the proof of Theorem 4

Lemma 10. Suppose that $(M, g)$ is self-dual. Then, if $U \in \mathcal{V}_{\sigma}$,

$$
\operatorname{Tr}_{k}(U)=\frac{t}{4} g(\mathcal{B}(U), \mathcal{B}(\sigma)), k=1,2
$$


Lemma 11. Suppose that $(M, g)$ is self-dual. Then, if $X \in T_{p} M, p=\pi(\sigma)$,

$$
\begin{aligned}
\operatorname{Tr}_{k}\left(X_{\sigma}^{h}\right)=\left[1+(-1)^{k}\right] \frac{s(p)}{144} X(s) & +\frac{1}{12}\left(\frac{t s(p)}{6}-2\right) X(s) \\
+ \text { Trace }_{h_{t}}\left\{\mathcal { V } _ { \sigma } \ni V \rightarrow \left[\frac{t}{8} g\left(\left(\nabla_{X} \mathcal{B}\right)(V), \mathcal{B}(V)\right)\right.\right. & \\
& \left.\left.+(-1)^{k+1} \frac{t s(p)}{24} g\left(\delta \mathcal{B}\left(K_{V} X\right), V\right)\right]\right\} .
\end{aligned}
$$

Sketch of the proof of Theorem 4. Suppose that $\mathcal{J}_{1}$ or $\mathcal{J}_{2}$ is a harmonic map. By Corollary $3(M, g)$ is self-dual or self-dual with constant scalar curvature. Moreover, $\operatorname{Tr}_{k}(U)=0$ for every vertical vector $U$, and $\operatorname{Tr}_{k}\left(X^{h}\right)=0$ for every horizontal vector $X^{h}, k=1$ or $k=2$. Note that in both cases the first term in the expression for $\operatorname{Tr}_{k}\left(X^{h}\right)$ given in Lemma 11 vanishes.

Lemma 10 implies that $\|\mathcal{B}(\cdot)\|^{2}=$ const on every fibre $\mathcal{Z}_{p}$ of the twistor space. One can show that this holds if and only if, at every point $p \in M$, at least three eigenvalues of the Ricci operator $\rho$ coincide. Then the next step in the proof is to demonstrate that the condition $\operatorname{Tr}_{k}\left(X_{\sigma}^{h}\right)=0$ for every $\sigma \in \mathcal{Z}, X \in T_{\pi(\sigma)} M$, is equivalent to the pair of identities

$$
g(\delta \mathcal{B}(X), \sigma)=0
$$

and

$$
\left(\frac{t s(p)}{144}+\frac{1}{6}\right) X(s)-\frac{t}{24} X\left(\|\rho\|^{2}\right)=0 .
$$

It is not hard to see that the identity (25) is equivalent to

$$
\text { Trace }\left\{E \rightarrow g\left(\left(\nabla_{E} \rho\right)\left(K_{\sigma} E\right), X\right)\right\}=0 .
$$

Let $r(X, Y)$ be the Ricci tensor and set

$$
d r(X, Y, Z)=\left(\nabla_{Y} r\right)(Z, X)-\left(\nabla_{Z} r\right)(Y, X) .
$$

Thus

$$
d r(X, Y, Z)=g\left(\left(\nabla_{Y} \rho\right)(Z), X\right)-g\left(\left(\nabla_{Z} \rho\right)(Y), X\right)
$$

Take an oriented orthonormal basis $\left(E_{1}, \ldots, E_{4}\right)$ such that $E_{2}=K_{\sigma} E_{1}$ and $E_{4}=$ $-K_{\sigma} E_{3}$. Then

$$
d r\left(X, E_{1}, E_{2}\right)-d r\left(X, E_{3}, E_{4}\right)=\sum_{m=1}^{4} g\left(\left(\nabla_{E_{m}} \rho\right)\left(K_{\sigma} E_{m}\right), X\right) .
$$

Denote by $W_{-}$the 4 -tensor corresponding to the operator $\mathcal{W}_{-}$,

$$
W_{-}(X, Y, Z, T)=g\left(\mathcal{W}_{-}(X \wedge Y), Z \wedge T\right) .
$$

By the differential Bianchi identity we have

$$
d r\left(X, E_{1}, E_{2}\right)-d r\left(X, E_{3}, E_{4}\right)=-2\left[\delta W_{-}\left(X, E_{1}, E_{2}\right)-\delta W_{-}\left(X, E_{3}, E_{4}\right)\right] .
$$

Since $(M, g)$ is self-dual, we see from the latter identity that identity (25) is always satisfied. Identity (27) shows also that

$$
d r(X, \sigma)=0, \quad \sigma \in \mathcal{Z}, \quad X \in T_{\pi(\sigma)} M .
$$

Let $\lambda_{1}(p) \leq \lambda_{2}(p) \leq \lambda_{3}(p) \leq \lambda_{4}(p)$ be the eigenvalues of the symmetric operator $\rho_{p}: T_{p} M \rightarrow T_{p} M$ in ascending order. It is well-known that the functions $\lambda_{1}, \ldots, \lambda_{4}$ are continuous (see, e.g. [23, Chapter Two, §5.7 ] or [30, Chapter I, §3]). We have seen that, at every point of $M$, at least three eigenvalues of the operator $\rho$ 
coincide. The set $U$ of points at which exactly three eigenvalues coincide is open by the continuity of $\lambda_{1}, \ldots, \lambda_{4}$. For every $p \in U$, denote the simple eigenvalue of $\rho_{p}$ by $\lambda(p)$ and the triple eigenvalue by $\mu(p)$, so the spectrum of $\rho$ is $(\lambda, \mu, \mu, \mu)$ with $\lambda(p) \neq \mu(p)$ for every $p \in U$. As is well-known, the implicit function theorem implies that the function $\lambda$ is smooth. Then the function $\mu=\frac{1}{3}(s-\lambda)$ is also smooth. It is also well-known that, in a neighbourhood of every point $p$ of $U$, there is a (smooth) unit vector field $E$ which is an eigenvector of $\rho$ corresponding to $\lambda$. (for a proof see [26, Chapter 9, Theorem 7]). Let $\alpha$ be the dual 1-form to $E$, $\alpha(X)=g(E, X)$. Then

$$
r(X, Y)=(\lambda-\mu) \alpha(X) \alpha(Y)-\mu g(X, Y)
$$

in a neighbourhood of $p$. Using this representation of the Ricci tensor, identity $\delta r=$ $-\frac{1}{2} d s$, and (28) one can prove that the scalar curvature $s$ is locally constant on $U$. Then identity (26) implies that $\|\rho\|^{2}$ is locally constant. Thus, in a neighbouhood of every point $p \in U$, we have $\lambda+3 \mu=a$ and $\lambda^{2}+3 \mu^{2}=b^{2}$, where $a$ and $b$ are some constants. It follows that $\mu=12^{-1}\left(3 a \pm \sqrt{12 b^{2}-3 a^{2}}\right)$. Note that $12 b^{2}-3 a^{2} \neq 0$, since otherwise we would have $\mu=\frac{1}{4} a$, hence $\lambda=a-3 \mu=\frac{1}{4} a=\mu$, a contradiction. Since $\mu$ is continuous, we see that $\mu$ is constant, hence $\lambda$ is also constant. Then one can show that the 1 -form $\alpha$ is parallel. It follows that the restriction of the Ricci tensor to $U$ is parallel.

In the interior of the closed set $M \backslash U$ the eigenvalues of the Ricci tensor coincide, hence the metric $g$ is Einstein on this open set. Therefore, the scalar curvature $s$ is locally constant on $\operatorname{Int}(M \backslash U)$ and the Ricci tensor is parallel on it. Thus, the Ricci tensor is parallel on the open set $U \cup \operatorname{Int}(M \backslash U)=M \backslash b U$, where $b U$ stands for the boundary of $U$. Since $M \backslash b U$ is dense in $M$, it follows that the Ricci tensor is parallel on $M$. This implies that the eigenvalues $\lambda_{1} \leq \ldots \leq \lambda_{4}$ of the Ricci tensor are constant. Thus, either $M$ is Einstein, or exactly three of the eigenvalues coincide. In the second case the simple eigenvalue $\lambda=0$ by [12, Lemma 1]. Therefore $M$ is locally the product of an interval in $\mathbb{R}$ and a 3-dimensional manifold of constant curvature.

Conversely, suppose that $(M, g)$ is self-dual and Einstein, or locally is the product of an interval and a manifold of constant curvature. Then at least three of the eigenvalues of the Ricci tensor coincide which, as we have noted, implies that $\|\mathcal{B}(\cdot)\|^{2}=$ const on every fibre of $\mathcal{Z}$. It follows that $g(\mathcal{B}(\sigma), \mathcal{B}(\tau))=0$ for every $\sigma, \tau \in \mathcal{Z}$ with $g(\sigma, \tau)=0$. Therefore, $T_{k}(U)=0$ for every vertical vector $U$, $k=1,2$, by Lemma 10. Moreover, $T_{k}\left(X^{h}\right)=0$ by Lemma [1] since the scalar curvature is constant and $\nabla \mathcal{B}=0$.

\section{Almost hermitian structures on 4-MANifolds that are harmonic MAPS}

Let $(M, g)$ be a Riemannian 4-manifold and $J$ a compatible almost complex structure on it. Henceforth in this section, we shall consider $M$ with the orientation induced by $J$, and the positive twistor space $\mathcal{Z}_{+}$will be denoted by $\mathcal{Z}$.

Denote the Ricci tensor of $(M, g)$ by $\rho$ and let $\rho^{*}$ be the $*$-Ricci tensor of the almost Hermitian manifold $(M, g, J)$. Recall that the latter is defined by

$$
\rho^{*}(X, Y)=\operatorname{trace}\{Z \rightarrow R(J Z, X) J Y\} .
$$


Denote by $N$ the Nijenhuis tensor of $J$

$$
N(Y, Z)=-[Y, Z]+[J Y, J Z]-J[Y, J Z]-J[J Y, Z] .
$$

It is well-known (and easy to check) that

$$
2 g\left(\left(\nabla_{X} J\right)(Y), Z\right)=d \Omega(X, Y, Z)-d \Omega(X, J Y, J Z)+g(N(Y, Z), J X),
$$

for all $X, Y, Z \in T M$.

8.1. The case of integrable $J$. Suppose that the almost complex structure $J$ is integrable. Denote by $B$ the vector field on $M$ dual to the Lee form $\theta=-\delta \Omega \circ J$ with respect to the metric $g$. Then (29) and the identity $d \Omega=\theta \wedge \Omega$ imply the following well-known formula

$$
2\left(\nabla_{X} J\right)(Y)=g(J X, Y) B-g(B, Y) J X+g(X, Y) J B-g(J B, Y) X .
$$

It follows that, considering $J$ as a section of the vector bundle $\Lambda_{+}^{2} T M$,

$$
\nabla_{X} J=\frac{1}{2}(J X \wedge B+X \wedge J B) .
$$

Using this formula and Proposition 2, one can prove the following.

Theorem 5. (10]) Suppose that the almost complex structure $J$ is integrable. Then the map $J:(M, g) \rightarrow\left(\mathcal{Z}, h_{t}\right)$ is harmonic if and only $d \theta$ is a $(1,1)$-form and $\rho(X, B)=\rho^{*}(X, B)$ for every $X \in T M$.

Corollary 5. The map $J:(M, g) \rightarrow\left(\mathcal{Z}, h_{t}\right)$ defined by an integrable almost Hermitian structure $J$ on $(M, g)$ is a harmonic section if and only if the 2 -form $d \theta$ is of type $(1,1)$.

Remark. The 2 -form $d \theta$ of a Hermitian surface $(M, g, J)$ is of type $(1,1)$ if and only if the $\star$-Ricci tensor $\rho^{*}$ is symmetric.

The map $J: M \rightarrow \mathcal{Z}$ is an imbedding and one can ask when this imbedding is minimal, i.e. when $J(M)$ is a minimal submanifold of $\left(\mathcal{Z}, h_{t}\right)$. Note that $J$ is minimal exactly when it is a harmonic map from $M$ endowed with the metric $J^{*} h_{t}$ into $\left(\mathcal{Z}, h_{t}\right)$.

If $\Pi$ is the second fundamental form of the submanifold $J(M)$, then $\Pi\left(J_{*} X, J_{*} Y\right)$ is the normal component of $\widetilde{\nabla} J_{*}(X, Y)$. In particular $J(M)$ is a minimal submanifold if and only if the normal component of Trace $\widetilde{\nabla} J_{*}$ vanishes.

Theorem 6. (10) Suppose that the almost complex structure $J$ is integrable. Then the map $J: M \rightarrow\left(\mathcal{Z}, h_{t}\right)$ is a minimal isometric imbedding if and only if $d \theta$ is a $(1,1)$ form and $\rho(X, B)=\rho^{*}(X, B)$ for every $X \perp\{B, J B\}$.

8.2. The case of symplectic $J$. Suppose that $(M, g, J)$ is almost Kähler (symplectic).

Denote by $\Lambda_{0}^{2} T M$ the subbundle of $\Lambda_{+}^{2} T M$ orthogonal to $J$ (thus $\Lambda_{0}^{2} T_{p} M=$ $\left.\mathcal{V}_{J(p)}\right)$. Under this notation we have the following.

Theorem 7. ([10]) Let $(M, g, J)$ be an almost Kähler 4-manifold. Then the map $J:(M, g) \rightarrow\left(\mathcal{Z}, h_{t}\right)$ is harmonic if and only if the $*$-Ricci tensor $\rho^{*}$ is symmetric and

$$
\text { Trace }\left\{\Lambda_{0}^{2} T M \ni \tau \rightarrow R(\tau)(N(\tau))\right\}=0 .
$$

The proof makes use of the Weitzenböck formula. 
Theorem 8. ([10]) Let $(M, g, J)$ be an almost Kähler four-manifold. Then the map $J: M \rightarrow\left(\mathcal{Z}, h_{t}\right)$ is a minimal isometric imbedding, if and only if the $\star$-Ricci tensor $\rho^{*}$ is symmetric, and for every $p \in M$

$$
\operatorname{Trace}\left\{\Lambda_{0}^{2} T_{p} M \ni \tau \rightarrow R_{p}(\tau)(N(\tau))\right\} \in \mathcal{N}_{p} .
$$

8.3. Examples. ([10])

Primary Kodaira surfaces. Every primary Kodaira surface $M$ can be obtained in the following way [25, p.787]. Let $\varphi_{k}(z, w)$ be the affine transformations of $\mathbb{C}^{2}$ given by

$$
\varphi_{k}(z, w)=\left(z+a_{k}, w+\bar{a}_{k} z+b_{k}\right),
$$

where $a_{k}, b_{k}, k=1,2,3,4$, are complex numbers such that

$$
a_{1}=a_{2}=0, \quad \operatorname{Im}\left(a_{3} \bar{a}_{4}\right)=m b_{1} \neq 0, \quad b_{2} \neq 0
$$

for some integer $m>0$. They generate a group $G$ of transformations acting freely and properly discontinuously on $\mathbb{C}^{2}$, and $M$ is the quotient space $\mathbb{C}^{2} / G$.

It is well-known that $M$ can also be described as the quotient of $\mathbb{C}^{2}$ endowed with a group structure by a discrete subgroup $\Gamma$. The multiplication on $\mathbb{C}^{2}$ is defined by

$$
(a, b) \cdot(z, w)=(z+a, w+\bar{a} z+b), \quad(a, b),(z, w) \in \mathbb{C}^{2},
$$

and $\Gamma$ is the subgroup generated by $\left(a_{k}, b_{k}\right), k=1, \ldots, 4$ (see, for example, [5]).

A frame of $\Gamma$-left-invariant vector fields on $\mathbb{C}^{2} \cong \mathbb{R}^{4}$ is given by

$$
A_{1}=\frac{\partial}{\partial x}-x \frac{\partial}{\partial u}+y \frac{\partial}{\partial v}, \quad A_{2}=\frac{\partial}{\partial y}-y \frac{\partial}{\partial u}-x \frac{\partial}{\partial v}, \quad A_{3}=\frac{\partial}{\partial u}, \quad A_{4}=\frac{\partial}{\partial v},
$$

where $x+i y=z, u+i v=w$. Let $g$ be the left-invariant Riemannian metric on $M \cong \mathbb{C}^{2} / \Gamma$ obtained from the metric on $\mathbb{C}^{2}$ for which the frame $A_{1}, \ldots, A_{4}$ is orthonormal.

It is a result by Hasegawa 21 that every complex structure on $M$ is induced by a left-invariant complex structure on $\mathbb{C}^{2}$. It is not hard to see $\left.(28,13]\right)$ that a left-invariant almost complex structure $J$ on $\mathbb{C}^{2}$ compatible with the metric $g$ is integrable if and only if it is given by

$$
J A_{1}=\varepsilon_{1} A_{2}, \quad J A_{3}=\varepsilon_{2} A_{4}, \quad \varepsilon_{1}, \varepsilon_{2}= \pm 1 .
$$

It is easy to check that, by Theorem 5 , the map $J:(M, g) \rightarrow\left(\mathcal{Z}, h_{t}\right)$ is harmonic.

It is also easy to give an explicit description of the twistor space $\left(\mathcal{Z}, h_{t}\right)([13])$, since $\Lambda_{+}^{2} M$ admits a global orthonormal frame defined by

$$
\begin{gathered}
s_{1}=\varepsilon_{1} A_{1} \wedge A_{2}+\varepsilon_{2} A_{3} \wedge A_{4}, \quad s_{2}=A_{1} \wedge A_{3}+\varepsilon_{1} \varepsilon_{2} A_{4} \wedge A_{2}, \\
s_{3}=\varepsilon_{2} A_{1} \wedge A_{4}+\varepsilon_{1} A_{2} \wedge A_{3} .
\end{gathered}
$$

Then we have a natural diffeomorphism $F: \mathcal{Z} \cong M \times S^{2}$ defined by $\left.\sum_{k=1}^{3} x_{k} s_{k}(p)\right) \rightarrow$ $\left(p, x_{1}, x_{2}, x_{3}\right)$ under which $J$ becomes the section $p \rightarrow(p, 1,0,0)$. Denote the pushforward of the metric $h_{t}$ under $F$ again by $h_{t}$. For $x=\left(x_{1}, x_{2}, x_{3}\right) \in S^{2}$, set

$u_{1}(x)=\varepsilon_{1} \varepsilon_{2}\left(-x_{3}, 0, x_{1}\right), \quad u_{2}(x)=\varepsilon_{2}\left(x_{2},-x_{1}, 0\right), u_{3}(x)=0, \quad u_{4}(x)=\varepsilon_{1}\left(0, x_{3},-x_{2}\right)$.

The differential $F_{*}$ sends the horizontal lifts $A_{i}^{h} i=1, \ldots, 4$, at a point $\sigma=$ $\sum_{k=1}^{3} x_{k} s_{k}(p) \in \mathcal{Z}$ to the vectors $A_{i}+u_{i}$ of $T M \oplus T S^{2}$. Then, if $X, Y \in T_{p} M$ and $P, Q \in T_{x} S^{2}$,

$$
\begin{gathered}
h_{t}(X+P, Y+Q)=g(X, Y) \\
+t<P-\sum_{i=1}^{4} g\left(X, A_{i}\right) u_{i}(x), Q-\sum_{j=1}^{4} g\left(Y, A_{j}\right) u_{j}(x)>
\end{gathered}
$$


where $<., .>$ is the standard metric of $\mathbb{R}^{3}$.

Now suppose again that $J$ is a left-invariant almost complex structure on $\mathbb{C}^{2}$ compatible with the metric $g$. Then the almost Hermitian structure $(g, J)$ is almost Kähler (symplectic) if and only if $J$ is given by $([28,13])$

$$
\begin{gathered}
J A_{1}=-\varepsilon_{1} \sin \varphi A_{3}+\varepsilon_{1} \varepsilon_{2} \cos \varphi A_{4}, \quad J A_{2}=-\cos \varphi A_{3}-\varepsilon_{2} \sin \varphi A_{4}, \\
J A_{3}=\varepsilon_{1} \sin \varphi A_{1}+\cos \varphi A_{2}, \quad J A_{4}=-\varepsilon_{1} \varepsilon_{2} \cos \varphi A_{1}+\varepsilon_{2} \sin \varphi A_{2}, \\
\varepsilon_{1}, \varepsilon_{2}= \pm 1, \varphi \in[0,2 \pi) .
\end{gathered}
$$

Suppose that $J$ is determined by these identities and set

$$
\begin{gathered}
E_{1}=A_{1}, \quad E_{2}=-\varepsilon_{1} \sin \varphi A_{3}+\varepsilon_{1} \varepsilon_{2} \cos \varphi A_{4}, \\
E_{3}=\cos \varphi A_{3}+\varepsilon_{2} \sin \varphi A_{4}, \quad E_{4}=A_{2} .
\end{gathered}
$$

Then $E_{1}, \ldots, E_{4}$ is an orthonormal frame of $T M$ for which $J E_{1}=E_{2}$ and $J E_{3}=E_{4}$. Define an orthonormal frame $s_{l}=s_{l}^{+}, l=1,2,3$, of $\Lambda_{+}^{2} T M$ by means of $E_{1}, \ldots, E_{4}$ via (2). Computing $\rho^{*}\left(E_{i}, E_{j}\right)$ one can see that the $*$-Ricci tensor is symmetric. Also, computing the curvature and the Nijenhuis tensor, we have

$$
\text { Trace }\left\{\Lambda_{0}^{2} T M \ni \tau \rightarrow R(\tau)(N(\tau))\right\}=R\left(s_{2}\right)\left(N\left(s_{2}\right)\right)+R\left(s_{3}\right)\left(N\left(s_{3}\right)\right)=0 .
$$

Thus, by Theorem 8 , $J$ defines a harmonic map.

As in the preceding case, it is easy to find an explicit description of the twistor space $\mathcal{Z}$ of $M$ and the metric $h_{t}([13])$. The frame $\left.\} s_{1}, s_{2}, s_{3}\right\}$ gives rise to an obvious diffeomeorphism $F: \mathcal{Z} \cong M \times S^{2}$ under which $J$ becomes the map $p \rightarrow(p, 1,0,0)$. The differential $F_{*}$ of this diffeomorphism sends the horizontal lifts $E_{i}^{h}, i=1, \ldots, 4$, to $E_{i}+u_{i}$ where

$$
\begin{gathered}
u_{1}(x)=\left(x_{3} \varepsilon_{1} \varepsilon_{2} \cos \varphi, x_{3} \varepsilon_{2} \sin \varphi,-x_{1} \varepsilon_{1} \varepsilon_{2} \cos \varphi-x_{2} \varepsilon_{2} \sin \varphi\right), \\
u_{2}(x)=\left(x_{2} \varepsilon_{1} \varepsilon_{2} \cos \varphi,-x_{1} \varepsilon_{1} \varepsilon_{2} \cos \varphi, 0\right), \quad u_{3}(x)=\left(x_{2} \varepsilon_{2} \sin \varphi,-x_{1} \varepsilon_{2} \sin \varphi, 0\right) \\
u_{4}(x)=\left(-x_{3} \varepsilon_{2} \sin \varphi, x_{3} \varepsilon_{1} \varepsilon_{2} \cos \varphi, x_{1} \varepsilon_{2} \sin \varphi-x_{2} \varepsilon_{1} \varepsilon_{2} \cos \varphi\right) .
\end{gathered}
$$

for $x=\left(x_{1}, x_{2}, x_{3}\right) \in S^{2}$. Then, if $X, Y \in T_{p} M$ and $P, Q \in T_{x} S^{2}$,

$$
\begin{gathered}
h_{t}(X+P, Y+Q)=g(X, Y) \\
+t<P-\sum_{i=1}^{4} g\left(X, E_{i}\right) u_{i}(x), Q-\sum_{j=1}^{4} g\left(Y, E_{j}\right) u_{j}(x)>.
\end{gathered}
$$

8.4. Four-dimensional Lie groups. By a result of A. Fino [17 for every leftinvariant almost Kähler structure $(g, J)$ with $J$-invariant Ricci tensor on a 4dimensional Lie group $M$ there exists an orthonormal frame of left-invariant vector fields $E_{1}, \ldots, E_{4}$ such that

$$
J E_{1}=E_{2}, \quad J E_{3}=E_{4}
$$

and

$$
\begin{gathered}
{\left[E_{1}, E_{2}\right]=0, \quad\left[E_{1}, E_{3}\right]=s E_{1}+\frac{s^{2}}{t} E_{2}, \quad\left[E_{1}, E_{4}\right]=\frac{s^{2}-t^{2}}{2 t} E_{1}-s E_{2}} \\
{\left[E_{2}, E_{3}\right]=-t E_{1}-s E_{2}, \quad\left[E_{2}, E_{4}\right]=-s E_{1}-\frac{s^{2}-t^{2}}{2 t} E_{2}, \quad\left[E_{3}, E_{4}\right]=-\frac{s^{2}+t^{2}}{t} E_{3}}
\end{gathered}
$$

where $s$ and $t \neq 0$ are real numbers. Using this table one can compute the $*$-Ricci and Nijenhius tensors. The computation shows that $J$ defines a harmonic map by virtue of Theorem 8 . 
8.5. Inoue surfaces of type $S^{0}$. Let us recall the construction of these surfaces (22]). Take a matrix $A \in S L(3, \mathbb{Z})$ with a real eigenvalue $\alpha>1$ and two complex eigenvalues $\beta$ and $\bar{\beta}, \beta \neq \bar{\beta}$. Choose eigenvectors $\left(a_{1}, a_{2}, a_{3}\right) \in \mathbb{R}^{3}$ and $\left(b_{1}, b_{2}, b_{3}\right) \in \mathbb{C}^{3}$ of $A$ corresponding to $\alpha$ and $\beta$, respectively. Then the vectors $\left(a_{1}, a_{2}, a_{3}\right),\left(b_{1}, b_{2}, b_{3}\right),\left(\overline{b_{1}}, \overline{b_{2}}, \overline{b_{3}}\right)$ are $\mathbb{C}$-linearly independent. Denote the upperhalf plane in $\mathbb{C}$ by $\mathbf{H}$ and let $\Gamma$ be the group of holomorphic automorphisms of $\mathbf{H} \times \mathbb{C}$ generated by

$$
g_{o}:(w, z) \rightarrow(\alpha w, \beta z), \quad g_{i}:(w, z) \rightarrow\left(w+a_{i}, z+b_{i}\right), i=1,2,3 .
$$

The group $\Gamma$ acts on $\mathbf{H} \times \mathbb{C}$ freely and properly discontinuously. Then $M=(\mathbf{H} \times$ $\mathbb{C}) / \Gamma$ is a complex surface known as the Inoue surface of type $S^{0}$. It has been shown by F. Tricerri 32 that every such a surface admits a locally conformal Kähler metric $g$ (cf. also [14]) obtained from the $\Gamma$-invariant Hermitian metric

$$
g=\frac{1}{v^{2}}(d u \otimes d u+d v \otimes d v)+v(d x \otimes d x+d y \otimes d y), \quad u+i v \in \mathbf{H}, \quad x+i y \in \mathbb{C} .
$$

on $\mathbf{H} \times \mathbb{C}$.

By Corollary $5:(M, g) \rightarrow\left(\mathcal{Z}, h_{t}\right)$ is a harmonic section. It is also a minimal isometric imbedding by Theorem 6 . However, $J$ is not a harmonic map according to Theorem 5

\section{REFERENCES}

[1] E. Abbena, An example of an almost Kähler manifold which is not Kählerian, Boll. Un. Mat. Ital. A(6) 3 (1984), 383-392.

[2] M. F. Atiyah, N. J. Hitchin, I. M. Singer, Self-duality in four-dimensional Riemannian geometry, Proc. Roy. Soc. London, Ser.A 362 (1978), 425-461.

[3] A. Besse, Einstein manifolds, Classics in Mathematics, Springer-Verlag, 2008.

[4] G. Bor, L. Hernández-Lamoneda, M. Salvai, Orthogonal almost-complex structures of minimal energy, Geom. Dedicata 127 (2007), 75-85.

[5] C. Borcea, Moduli for Kodaira surfaces, Composition Math. 52 (1984), 373-380.

[6] E. Calabi, H. Gluck, What are the best almost-complex structures on the 6-sphere?, Proc. Sym. Pure Math. 54 (1993), part 2, 99-106.

[7] J. Davidov, Einstein condition and twistor spaces of compatible partially complex structures, Diff. Geom. and its Appl. 22 (2005), 159-179

[8] J. Davidov, O. Mushkarov, On the Riemannian curvature of a twistor space, Acta Math. Hungarica 58 (1991), 319-332.

[9] J. Davidov, O. Mushkarov, Harmonic almost-complex structures on twistor spaces, Israel J. Math. 131 (2002), 319-332.

[10] J. Davidov, A. Ul Haq, O. Mushkarov, Almost complex structures that are harmonic maps, arXiv:1504.01610v2 [math.DG] 19 Aug 2015.

[11] J. Davidov, O. Mushkarov, Harmonicity of the Atiyah-Hitchin-Singer and Eells-Salamon almost complex structures, in preparation.

[12] J. Davidov, G. Grantcharov, O. Mushkarov, Twistorial examples of *-Einstein manifolds, Ann. Glob. Anal. Geom. 20 (2001), 103-115.

[13] J. Davidov, Twistorial construction of minimal hypersurfaces, Inter. J. Geom. Methods in Modern Physics 11, No 6 (2014), 1459964.

[14] S. Dragomir, L. Ornea, Locally conformal Kähler geometry, Progress in Math., v. 155, Birkhäuser, Boston-Basel-Berlin, 1998.

[15] J. Eells, L. Lemaire, Selected topics in harmonic maps, Cbms Regional Conference Series in Mathematics, vol. 50, AMS, Providernce, Rhode Island, 1983.

[16] J. Eells, S. Salamon, Twistorial constructions of harmonic maps of surfaces into fourmanifolds, Ann. Scuola Norm. Sup. Pisa, ser.IV, 12 (1985), 589-640.

[17] A. Fino, Almost Kähler 4-dimensional Lie groups with J-invariant Ricci tensor, Diff. Geom. Appl. 23 (2005), 26-37. 
[18] A. Gray, Minimal varieties and almost Hermitian manifolds, Michigan Math. J. 12 (1965), 273-287.

[19] A. Gray, L. M. Hervella, The sixteen classes of almost Hermitian manifolds and their linear invariants, Ann. Mat. Pure Appl. 123 (1980), 288-294.

[20] D. Gromol, W. Klingenberg, W. Meyer, Riemannsche Geometrie in Grossen, Lecture Notes in Mathematics vol. 55, Springer-Verlag, 1968.

[21] K. Hasegawa, Complex and Kähler structures on compact solvmanifolds, J. Symplectic Geom. 3 (2005), 749-767.

[22] M. Inoue, On surfaces of class VII $I_{0}$, Invent. Math. 24 (1974), 269-310.

[23] T. Kato, Perturbation theory for linear operators, Springer-Verlag, Berlin-Heidelberg-New York, 1980.

[24] I. Kim, Almost Kähler anti-self-dual metrics, Ph.D. thesis, Stony Brook University, May 2014, available at www.math.stonybrook.edu/alumni/2014-Inyong-Kim.pdf; see also arXiv:1511.07656v1 [math.DG] 24 Nov 2015.

[25] K. Kodaira, On the structure of compact complex analytic surfaces I. Amer. J. Math. 86 (1964), 751-798.

[26] P. Lax, Linear algebra and its applications, John \& Sons, Inc., Hoboken, New Jersey, 2007.

[27] C. LeBrun, Anti-self-dual hermitian metrics on blow-up Hopf surfaces, Math. Ann. 289 (1991), 383-392.

[28] O. Muškarov, Two remarks on Thurston's example, In: Complex analysis and applications '85 (Varna, 1985), Publ. House Bulgar. Acad. Sci., Sofia, 1986, pp. 461-468.

[29] O. Muškarov, Structures presque hermitienes sur espaces twistoriels et leur types, C. R. Acad. Sci. Paris Sér.I Math. 305 (1987), 307-309.

[30] F. Rellich, Perturbation theory of eigenvalue problems, Notes on mathematics and its applications, Gordon and Breach science publishers, New York-London-Paris, 1969.

[31] I. M. Singer, J. A. Thorpe, The curvature of 4-dimensional Einstein spaces, in papers in Honor of K. Kodaira, Princeton University Press (Princeton), 1969, pp. 355-365.

[32] F. Tricerri, Some example of locally conformal Kähler manifolds, Rend. Sem. Mat. Univ. Torino 40 (19982), 81-92.

[33] W. P. Thurston, Some simple examples of symplectic manifolds, Proc. Amer. Math. Soc. 55 (1976), 467-468.

[34] J. Vilms, Totally geodesic maps, J. Diff. Geom. 4 (1970), 73-79.

[35] C. M. Wood, Instability of the nearly-Kähler six-sphere, J. reine angew. Math. 439 (1993), 205-212.

[36] C. M. Wood, Harmonic almost-complex structures, Compositio Mathematica 99 (1995), 183-212.

Johann Davidov, Institute of Mathematics and Informatics, Bulgarian Academy of Sciences, Acad. G.Bonchev Str. Bl.8, 1113 Sofia, Bulgaria,

AND

University of Structural Engineering and Architecture "L.Karavelov", 175 SuhodolSka St., 1373 Sofia, Bulgaria 